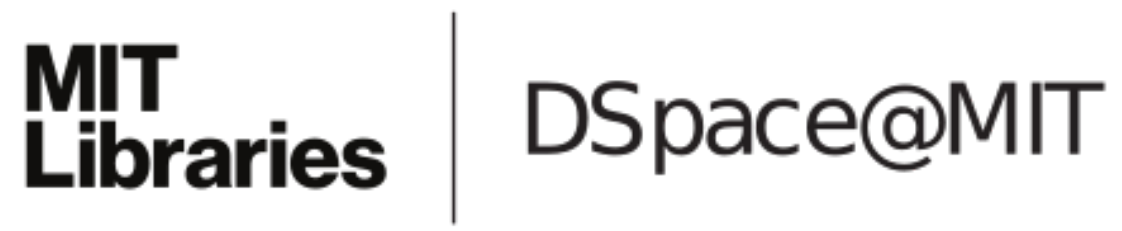

\author{
MIT Open Access Articles
}

\section{Quantum phases of hard-core dipolar bosons in coupled one-dimensional optical lattices}

The MIT Faculty has made this article openly available. Please share how this access benefits you. Your story matters.

Citation: Safavi-Naini, A., B. Capogrosso-Sansone, and A. Kuklov. "Quantum phases of hard-core dipolar bosons in coupled one-dimensional optical lattices." Phys. Rev. A 90, 043604 lOctober 2014). (c) 2014 American Physical Society

As Published: http://dx.doi.org/10.1103/PhysRevA.90.043604

Publisher: American Physical Society

Persistent URL: http://hdl.handle.net/1721.1/90840

Version: Final published version: final published article, as it appeared in a journal, conference proceedings, or other formally published context

Terms of Use: Article is made available in accordance with the publisher's policy and may be subject to US copyright law. Please refer to the publisher's site for terms of use. 


\title{
Quantum phases of hard-core dipolar bosons in coupled one-dimensional optical lattices
}

\author{
A. Safavi-Naini, ${ }^{1,2}$ B. Capogrosso-Sansone, ${ }^{3}$ and A. Kuklov ${ }^{4}$ \\ ${ }^{1}$ Department of Physics, Massachusetts Institute of Technology, Cambridge, Massachusetts 02139, USA \\ ${ }^{2}$ ITAMP, Harvard-Smithsonian Center for Astrophysics, Cambridge, Massachusetts 02138, USA \\ ${ }^{3}$ Homer L. Dodge Department of Physics and Astronomy, The University of Oklahoma, Norman, Oklahoma 73019, USA \\ ${ }^{4}$ Department of Engineering \& Physics, CSI, CUNY, Staten Island, New York 10314, USA
}

(Received 27 May 2014; published 6 October 2014)

\begin{abstract}
Hard-core dipolar bosons trapped in a parallel stack of $N \geqslant 2$ one-dimensional optical lattices (tubes) can develop several phases made of composites of particles from different tubes: superfluids, supercounterfluids, and insulators as well as mixtures of those. Bosonization analysis shows that these phases are thresholdless with respect to the dipolar interaction, with the key "control knob" being filling factors in each tube, provided the intertube tunneling is suppressed. The effective $a b$ initio quantum Monte Carlo algorithm capturing these phases is introduced and some results are presented.
\end{abstract}

DOI: 10.1103/PhysRevA.90.043604

PACS number(s): $67.85 . \mathrm{Hj}$

\section{INTRODUCTION}

The unprecedented level of control in ultracold atom experiments, and the flexibility offered by these systems allows for the manipulation of the interparticle interactions, as well as the creation of a variety of lattice geometries [1]. Besides providing a perfect setup for the realization of paradigmatic condensed matter models $[2,3]$, these ultracold systems are a playground for studying a variety of novel quantum phases. Mentioning just a few, these are supercounterfluids (SCF) [4], paired superfluids [5], and superfluids of molecular chains [6]. Recent experimental success in trapping ultracold bosonic atomic mixtures [3,7-9] has rendered the study of pairing between components very timely. The impressively rapid experimental progress towards controlling polar molecules [10-18] gives hope for accessing quantum many-body systems with long-range and anisotropic interaction in the very near future [18].

A prominent example of bosonic systems currently available experimentally consists of an array of coupled onedimensional (1D) tubes, with the interaction between tubes provided by dipolar forces. In the hard-core limit, this system represents coupled spin chains, which is one of the central topics in low-dimensional condensed matter physics [19]. In the absence of intertube tunneling, this system is formally equivalent to multicomponent mixtures of noncovertable atomic species, where several predictions for the novel phases have been made [4,5]. An interesting opportunity for even more exotic superfluids in the $N=2$ setup-consisting of $p \geqslant 1$ molecules from one tube and $q>1$ ones form the other- has been suggested in Refs. [20,21]. Physics of the multitube setup becomes much richer when the intertube tunneling is finite: As proposed in Refs. [22,23], the transition point in the $N$-tube setup features the emergence of $Z_{N}$ parafermions.

In the present work we discuss possible phases in a system of hard-core bosons confined to a stack of $N$ one-dimensional lattices-tubes (see Fig. 1). Bosons in neighboring tubes interact via intertube interaction (either nearest-neighbor or dipole-dipole), with the intertube tunneling suppressed (this can be achieved experimentally with a deep optical lattice potential along the direction perpendicular to the tubes). Our focus is on quantum many-body phases of self-assembled chains of molecules from different tubes [6,24-29]. Most previous theoretical studies of the many-body phases of dipolar bosons confined to a stack of one-dimensional tubes employ variational methods. In Ref. [30], a quantum to classical mapping in the discretized time representation has been used. The resulting model is amenable to classical Monte Carlo technique, and it has been shown that bosons in a stack of one-dimensional tubes can form superfluids of strongly interacting multiatomic complexes-chain superfluids (CSF), each chain consisting of one molecule from each tube, and there is, in general, a threshold for the CSF formation.

In what follows we will use bosonization and renormalization group (RG) techniques along with Monte Carlo simulations to study the quantum phases of hard-core bosons trapped in a multitube geometry. In contrast to what was previously done numerically in Ref. [30], here we study the actual quantum Hamiltonian of hard-core bosons by means of the $a b$ initio path integral quantum Monte Carlo (QMC) simulations (in continuous time) using a multiworm algorithm [30] — an extension of the worm algorithm [31] and its two-worm modification [5].

Our findings can be summarized as follows. Bosonization and $\mathrm{RG}$ predict the existence of interesting quantum phases such as CSF, supercounterfluid (SCF), and checker-board (CB) solid provided certain requirements on the filling factor in the tubes are fulfilled. These phases are predicted to form at an arbitrarily small intertube interaction between particles in the thermodynamical limit. For example, in the case of a dipolar gas with dipoles aligned perpendicular to the tubes and lying in the plane of the tubes, an arbitrarily small dipolar interaction is sufficient to destabilize independent superfluids in each tube in favor of the CSF phase, provided the filling factors in each tube are the same. We use QMC to explicitly demonstrate this property for the case of two tubes. We also perform simulations for the case of three layers and explicitly demonstrate the existence of CSF and CB solid. The numerical results for the CB solid support the thresholdless nature of this phase as well.

Our study is a first step toward $a b$ initio simulations of more involved cases including spin ladders and polar molecules with intertube tunneling. Using this algorithm it 


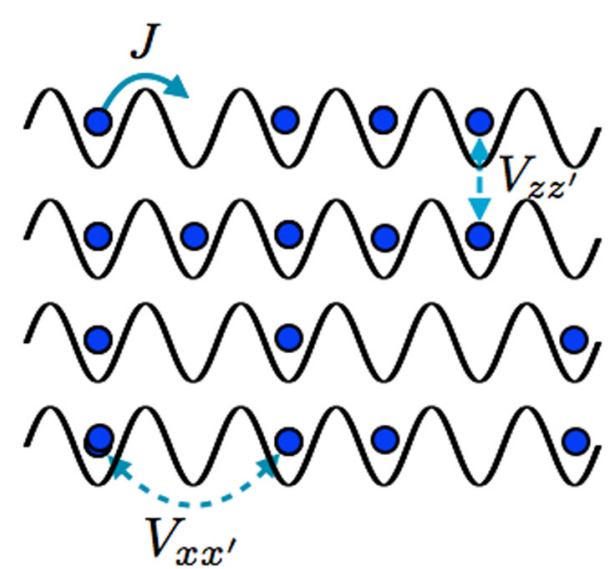

FIG. 1. (Color online) A sketch of the system studied: hard-core bosons (solid circles) can tunnel (as marked by solid arrow) along the tubes-1D optical lattices ( $N=4$ of them are shown); dashed arrows indicate the intraplane $V_{x x^{\prime}}$ and the interplane interactions $V_{z z^{\prime}}$.

should be possible to provide accurate recommendations for the experimental realizations of the complex dipolar phases.

This paper is organized as follows. In Sec. II we present the system Hamiltonian for arbitrary two-body density-density interaction and discuss specific cases of dipolar interaction for two different polarizations of the dipoles. In Sec. III we present a description of the system in terms of the linear response theory and discuss the properties of the phases which can be stabilized in this geometry: $N$-atomic superfluids, composite superfluids, supercounterfluids, and composite insulators. In Sec. IV the system is discussed in terms of the bosonized action and RG for the composite superfluids, supercounterfluids, and the composite insulator at half-integer filling factor. In Sec. V we describe the multiworm algorithm used and present numerical evidence for the thresholdless nature of composite superfluids for the case of two layers. We also numerically demonstrate the existence of the composite superfluid phase and composite insulating phase at half filling for the case of three layers. Finally, we conclude in Sec. VI.

\section{HAMILTONIAN}

The system under consideration is described by the singleband tight-binding Hamiltonian,

$$
\begin{aligned}
H= & -J \sum_{\left\langle x, x^{\prime}\right\rangle, z} a_{x z}^{\dagger} a_{x^{\prime} z} \\
& +\frac{1}{2} \sum_{x z ; x^{\prime} z^{\prime}} V\left(x-x^{\prime}, z-z^{\prime}\right) n_{x z} n_{x^{\prime} z^{\prime}}-\sum_{x z} \mu_{z} n_{x z},
\end{aligned}
$$

in grand canonical ensemble. Here $J>0$ stands for the intratube tunneling amplitude, $a_{x z}^{\dagger}\left(a_{x z}\right)$ is the creation (annihilation) operator for a hard-core boson at site $(x, z)$, where the second index $z=0,1,2, \ldots, N-1$ labels the tubes and the first one $x=0,1,2, \ldots, L$ is the coordinate along a tube. Here, \langle\rangle denotes summation over nearest neighbors, $n_{x z}=a_{x z}^{\dagger} a_{x z}$, and $\mu_{z}$ is the chemical potential, which can be different in different tubes.
The interaction $V(x, z)$ can be arbitrary. In the case of the dipole-dipole interaction, with the polarization axis being perpendicular to the tubes and belonging to the plane of the tubes, it takes the form,

$$
V(x, z)=V_{d} \frac{x^{2}-2 z^{2}}{\left(x^{2}+z^{2}\right)^{5 / 2}},
$$

where $V_{d}>0$ sets the energy scale. In this geometry, the interaction along the $z$ axis is attractive. As we will discuss below, in $1 \mathrm{D}$, arbitrary small $V_{d}$ can induce superfluidity of quasimolecular complexes. This result follows from the bosonization analysis and has previously been noted for the case of pairing (that is, $N=2$ ) of hard-core bosons in Ref. [32].

The repulsive part of the interaction along the $x$ axis favors solidification. A special role is played by the filling factor $v=$ $1 / 2$. As we will show later, in the case $N>2$ the insulating phase featuring 1D checkerboard order emerges in the limit $V_{d} \rightarrow 0$ even if no intralayer repulsion is explicitly introduced.

When dipoles are polarized perpendicularly to the tubes plane, the interaction becomes purely repulsive,

$$
V(x, z)=V_{d} \frac{1}{\left(x^{2}+z^{2}\right)^{3 / 2}},
$$

and can result in SCF [4] phases which are also thresholdless with respect to the interaction.

\section{DENSITY CONTROLLED QUANTUM PHASES IN LAYERED SYSTEMS}

A system of hard-core bosons, trapped in one-dimensional tubes with no intertube Josephson coupling, forms $N$ independent superfluids characterized by $N$ quasicondensate order parameters $\left\langle\psi_{z}\right\rangle \sim \exp \left(i \phi_{z}\right)$ with phases $\phi_{z}, z=0,1,2, \ldots N-$ 1. The hard-core nature of bosons in each tube plays a special role. As we will see below, an arbitrary small intertube interaction can induce multiplicity of various superfluid and insulating phases depending on the filling factors $v_{z}$ in the tubes. The counterintuitive thresholdless nature of the phases simply means that observing them is possible for arbitrary small $V_{d}$ on correspondingly large spatial scales. It is worth noting that, depending on a combination of the filling factors, various types of mixtures of such phases can exist as well.

\section{A. Thouless phase twists and windings}

Here we introduce a description in terms of the generalized superfluid stiffness $R_{z z^{\prime}}$ and superfluid compressibility $C_{z z^{\prime}}$. This language of the generalized superfluid response turns out to be very helpful in defining ground states of the bosonic complexes as well as in characterizing ground states numerically. The response matrices are defined through contributions to the system action as a result of imposing infinitesimal Thouless phase twists $\vec{\phi}^{\prime}(z)=\left(\phi_{x}^{\prime}(z), \phi_{\tau}^{\prime}(z)\right)$ on the space-time boundaries of the tubes. Such twists can be viewed in terms of the corresponding gauge potentials $A_{x}(z, x)=\phi_{x}^{\prime}(z) / L$ along space and $A_{\tau}(z, \tau)=\phi_{\tau}^{\prime}(z) / \beta$ along time, where $L, \beta$ stand for tubes length and inverse temperature in atomic units, respectively. It is important that, in the case of the 
periodic boundary conditions on the phases $\phi_{z}(x, \tau)$ of the fields, such gauge potentials cannot be absorbed into the phases.

In general, the infinitesimal contribution of the twists to the action is given by

$$
E=\sum_{z z^{\prime}}\left[\frac{\beta}{2 L} R_{z z^{\prime}} \phi_{x}^{\prime}(z) \phi_{x}^{\prime}\left(z^{\prime}\right)+\frac{L}{2 \beta} C_{z z^{\prime}} \phi_{\tau}^{\prime}(z) \phi_{\tau}^{\prime}\left(z^{\prime}\right)\right] .
$$

The quantities $R_{z z^{\prime}}$ and $C_{z z^{\prime}}$ can be expressed in terms of topological properties of the particle world lines, windings $\vec{W}(z)=\left[W_{x}(z), W_{\tau}(z)\right]$, and can be measured numerically. Global gauge invariance of the system implies that the total partition function $Z=\operatorname{Tr}[\exp (-\beta H)]$ can be represented as a statistical sum over all possible winding numbers of closed world lines of particles as

$$
Z=\sum_{\{\vec{W}(z)\}} Z[\{\vec{W}\}] \exp \left[i \sum_{z} \vec{W}(z) \vec{\phi}^{\prime}(z)\right],
$$

where $Z[\{\vec{W}\}]$ stands for a functional of windings in all tubes. The superfluid stiffnesses can be obtained as second derivatives of $E=-\ln Z$ with respect to $\left[\phi_{x}^{\prime}(z), \phi_{\tau}^{\prime}(z)\right]$ in the limit $\vec{\phi}^{\prime}(z) \rightarrow 0$ as

$$
\begin{aligned}
& R_{z z^{\prime}}=\frac{L}{\beta}\left[\left\langle W_{x}(z) W_{x}\left(z^{\prime}\right)\right\rangle-\left\langle W_{x}(z)\right\rangle\left\langle W_{x}\left(z^{\prime}\right)\right\rangle\right], \\
& C_{z z^{\prime}}=\frac{\beta}{L}\left[\left\langle W_{\tau}(z) W_{\tau}\left(z^{\prime}\right)\right\rangle-\left\langle W_{\tau}(z)\right\rangle\left\langle W_{\tau}\left(z^{\prime}\right)\right\rangle\right] .
\end{aligned}
$$

As long as the tubes are identical, $R_{z z^{\prime}}$ and $C_{z z^{\prime}}$ depend on the difference $z-z^{\prime}, R_{z z^{\prime}}=R\left(z-z^{\prime}\right)$, and $C_{z z^{\prime}}=C\left(z-z^{\prime}\right)$. Hence, the Fourier transform along the $z$ axis can be used, $\tilde{R}\left(q_{z}\right)=\sum_{z} R(z) \exp \left(i q_{z} z\right), \tilde{C}\left(q_{z}\right)=\sum_{z} C(z) \exp \left(i q_{z} z\right)$, where $\quad q_{z}=2 \pi m_{z} / N, m_{z}=0,1,2, \ldots, N-1$. Then, Eq. (5) expressed in terms of the Fourier transforms $\tilde{\phi}_{x, \tau}^{\prime}\left(q_{z}\right)=N^{-1 / 2} \sum_{z} \phi_{x, \tau}^{\prime}(z) \exp \left(i q_{z} z\right), \tilde{W}_{x, \tau}\left(q_{z}\right)=$ $N^{-1 / 2} \sum_{z} W_{x, \tau}(z) \exp \left(i q_{z} z\right)$ gives

$$
\tilde{R}\left(q_{z}\right)=\frac{L}{\beta}\left[\left\langle\tilde{W}_{x}\left(q_{z}\right) \tilde{W}_{x}\left(-q_{z}\right)\right\rangle-\left\langle\tilde{W}_{x}\left(q_{z}\right)\right\rangle\left\langle\tilde{W}_{x}\left(-q_{z}\right)\right\rangle\right],
$$

and

$$
\tilde{C}\left(q_{z}\right)=\frac{\beta}{L}\left[\left\langle\tilde{W}_{\tau}\left(q_{z}\right) \tilde{W}_{\tau}\left(-q_{z}\right)\right\rangle-\left\langle\tilde{W}_{\tau}\left(q_{z}\right)\right\rangle\left\langle\tilde{W}_{\tau}\left(-q_{z}\right)\right\rangle\right] .
$$

These equations represent an extension of the Ceperley and Pollock expression [33] for the superfluid stiffness and compressibility.

In full analogy with the case $N=1$, the ratio $V_{s}\left(q_{z}\right)=$ $\sqrt{\tilde{R}\left(q_{z}\right) / \tilde{C}\left(q_{z}\right)}$ has the meaning of the speed of sound propagating along tubes with dispersion along the $z$ axis. Extending the analogy, the product $\tilde{R}\left(q_{z}\right) \tilde{C}\left(q_{z}\right)$ gives the Luttinger "parameter" (rather, Luttinger matrix) as

$$
\tilde{K}\left(q_{z}\right)=\pi \sqrt{\tilde{R}\left(q_{z}\right) \tilde{C}\left(q_{z}\right)} .
$$

Thus, the action for arbitrary (small) phase fluctuations of the translationally invariant (along the $z$ axis) system renormalized by the interactions becomes

$$
\begin{aligned}
S_{R}= & \int_{0}^{\beta} d \tau \int d x \sum_{q_{z}}\left[\frac{V_{s}\left(q_{z}\right) \tilde{K}\left(q_{z}\right)}{2 \pi}\left|\nabla_{x} \tilde{\phi}_{q_{z}}(x, \tau)\right|^{2}\right. \\
& \left.+\frac{\tilde{K}\left(q_{z}\right)}{2 \pi V_{s}\left(q_{z}\right)}\left|\nabla_{\tau} \tilde{\phi}_{q_{z}}(x, \tau)\right|^{2}\right],
\end{aligned}
$$

where $\tilde{\phi}_{q_{z}}(x, \tau)$ are the Fourier components of the phases $\phi_{z}(x, \tau)$ with respect to the tube index $z$.

The speed of sound is not significantly renormalized compared to the strong renormalization of superfluid stiffness $R$ and compressibility $C$ [32]. This simply means that the space-time symmetry of the superfluid-insulator transitions is preserved in the translationally invariant system. Thus, for all practical purposes, the dispersion of the speed of sound $V_{s}$ vs $q_{z}$ can be ignored so that $R_{z z^{\prime}}=C_{z z^{\prime}}$ in units of $V_{s}=1$. In this limit, the Luttinger matrix and the matrix of stiffnesses are equal to each other. Then, the generalized linear response can be fully described by the following translationally invariant action $S_{R}=\int_{0}^{\beta} d \tau \int d x \sum_{q_{z}} \frac{1}{2 \pi} \tilde{K}\left(q_{z}\right)\left|\vec{\nabla} \tilde{\phi}\left(q_{z}\right)\right|^{2}$, or in the direct $z$ space as

$$
S_{R}=\int_{0}^{\beta} d \tau \int d x \sum_{z, z^{\prime}} \frac{1}{2 \pi}(\hat{K})_{z, z^{\prime}} \vec{\nabla} \phi_{z} \vec{\nabla} \phi_{z^{\prime}}
$$

where $\hat{K}$ stands for the Luttinger parameter matrix with the dimension $N \times N$. It is worth mentioning that this form features the nonviscous drag between superfluid flows in different tubes. It is responsible for the formation of the complex superfluid and supercounterfluid phases. We will be referring to the form (12) and, specifically, to the properties of the Luttinger matrix $\hat{K}$ while identifying the ground states of the bosonic complexes.

\section{B. $N$ atomic superfluids}

If the matrix $\hat{K}$ in (12) is nondegenerate (in the case when all filling factors are different and not complimentary to unity), there exists the standard algebraic (or 1D superfluid) order in the correlators $\left\langle\exp \left[i \phi_{z}(x)\right] \exp \left[-i \phi_{z}(0)\right]\right\rangle \sim 1 /|x|^{\left(\hat{K}^{-1}\right)_{z z} / 2}$ $\left\{\right.$ and $\left\langle\exp \left[i \phi_{z}(x)\right] \exp \left[-i \phi_{z^{\prime}}(0)\right]\right\rangle=0$ for $\left.z \neq z^{\prime}\right\}$. A sketch of this phase is shown in Fig. 2. As we will see below, should some filling factors become the same or complimentary to

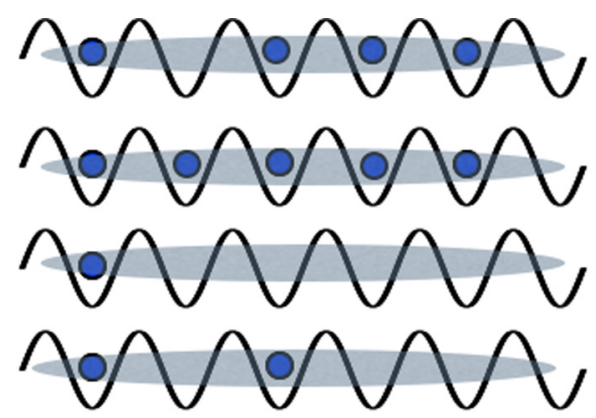

FIG. 2. (Color online) A sketch of the $N$-atomic superfluids characterized by $N$ independent off-diagonal algebraic orders (depicted by $N=4$ fuzzy clouds). 


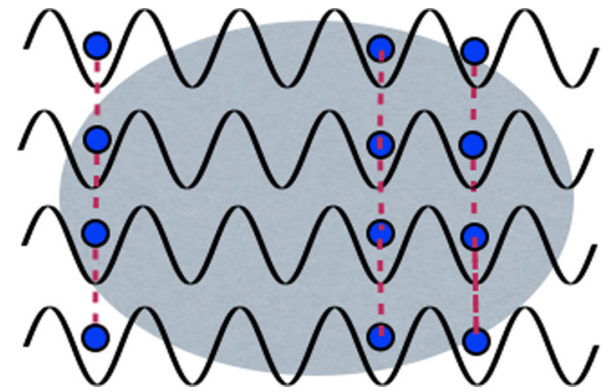

FIG. 3. (Color online) A sketch of the CSF phase. Dashed lines indicate binding of atoms from different tubes and the fuzzy cloud depicts single off-diagonal algebraic order for all tubes.

unity, the intertube interaction can easily destroy such atomic orders in favor of composite superfluids or supercounterfluids.

\section{Composite superfluids}

If all $N$ tubes are characterized by the same incommensurate filling factor $v$, the nature of the superfluid correlations changes dramatically as long as there is an arbitrary small attraction $V_{d}$ between the tubes. Specifically, the matrix $\hat{K}$ becomes degenerate so that $\left\langle\exp \left[i \phi_{z}(x)\right] \exp \left[-i \phi_{z}(0)\right]\right\rangle$ decays exponentially. The algebraic decay will be observed only in the $N$-body density matrix $\left\langle\Psi^{\dagger}(x) \Psi(0)\right\rangle$ where $\Psi(x)=$ $\psi_{z=0}(x) \psi_{z=1}(x) \ldots \psi_{z=N}(x)$. In other words, the matrix elements of $\hat{K}$ in Eq. (12) become all identical to each other, so that $N-1$ eigenvalues are equal to zero and only one remains finite-corresponding to a finite superfluid stiffness of the sum of the phases $\Phi=\sum_{z} \phi_{z}$. In terms of the Fourier components of the matrix kernel, $\tilde{K}\left(q_{z}=0\right) \neq 0$ while $\tilde{K}\left(q_{z} \neq 0\right)=0$. This defines the CSF, a superfluid of quasimolecular complexes, each complex consisting of $N$ bosons-one from each tube. A sketch of this phase is shown in Fig. 3.

If $N>2$, it is possible to have a situation when only $1<M<N$ tubes have identical filling factors. Then, the composite superfluid will be formed among these tubes while others carry the standard atomic superfluids. In general, a group of $M$ layers with the same filling factor adds degree of degeneracy $M-1$ to the matrix $\hat{K}$. In other words, the number of the remaining superfluid phases is equal to $N$ minus the total degree of degeneracy. This means that the matrix $\hat{K}$ will have as many zero eigenvalues as there exist restored $U(1)$ symmetries.

\section{Supercounterfluids}

The concept of supercounterfluidity (SCF) has been introduced for two-component systems in Ref. [4]. SCF can exist in a lattice when the filling factors $v_{1}$ and $v_{2}$ for both components complement each other to an integer filling, $v_{1}+v_{2}=1$. Then, the repulsive interaction can induce binding of atoms of sort " 1 " to holes of sort " 2 ." Using the language of broken symmetries, the $U(1) \times U(1)$ symmetry becomes partially restored so that only one $U(1)$ symmetry remains broken. In terms of the fields, the field $\exp \left(i \phi_{1}\right) \exp \left(-i \phi_{2}\right)$ is condensed while $\exp \left(i \phi_{1}\right) \exp \left(i \phi_{2}\right)$ becomes disordered. Accordingly, the

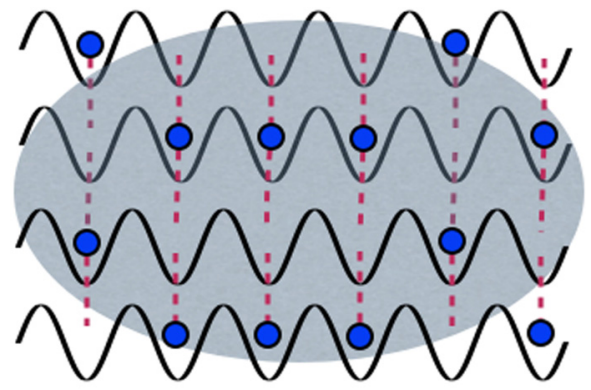

FIG. 4. (Color online) A sketch of the SCF phase in $N>2$ tubes. Similarly to Fig. 3, the fuzzy cloud depicts a single off-diagonal algebraic order for all tubes. The dashed lines indicate binding between atoms and holes in the tubes with complementary fillings and between atoms in the tubes with the same fillings.

superflow can only exist in the counterflow manner-when transfer of one atom of sort " 1 " is compensated by motion of one atom of sort " 2 " in the opposite direction.

This property can naturally be extended to a general case of $N$ sorts of atoms when the superflow of, say, $M<N$ components is (partially) compensated by the counterflow of the remaining components. The SCF phase is sketched in Fig. 4.

In general, there could be $M_{1}$ tubes all with the same filling factors $v_{1}$ and $M_{2}$ tubes also with identical filling factors $v_{2}=1-v_{1}$ so that $v_{2} \neq v_{1}$. Thus, there are two groups of the composite superfluids, consisting of $M_{1}$ and $M_{2}$ complexes. Accordingly, there are $M_{1}-1+M_{2}-1$ restored symmetries. Moreover, the backscattering (BS) interaction between particles from the first and the second groups restores one additional symmetry. The corresponding composite operator which characterizes the algebraic order is $\Phi_{M_{1}, M_{2}}(x)=$ $\psi_{z_{1}}(x) \ldots \psi_{z_{M_{1}}}(x) \psi_{z_{1}^{\prime}}^{\dagger}(x) \ldots \psi_{z_{M_{2}}^{\prime}}^{\dagger}(x)$, where $z_{1}, z_{2}, \ldots, z_{M_{1}}$ label tubes from the first group and $z_{1}^{\prime}, z_{2}^{\prime}, \ldots, z_{M_{2}}^{\prime}$-from the second. In such a phase a transfer of $M_{1}$ atoms from the first group is compensated by the countermotion of $M_{2}$ atoms from the second group, so that there is a net transfer of $M_{1}-M_{2}$ atoms. Accordingly, $M_{1}+M_{2}-1$ eigenvalues of the matrix $\hat{K}$ in Eq. (12) are zero. In other words, the resulting state can be thought of as a bound state of two composite superfluids in the counterflow regime - a natural generalization of the two-component SCF [4].

In the special case when all, e.g, odd tubes have filling factor $v$ and all even ones have $1-v$ (as exemplified in Fig. 4), the Fourier transform can be used. In this case all Fourier harmonics but $\tilde{K}\left(q_{z}=\pi\right)$ are equal to zero, so that there is no net transfer of atoms. As we will discuss below, such a phase can also be realized for arbitrary small $V_{d}$.

\section{E. Composite insulators}

The easiest way to form an insulator in 1D lattices is at filling factor $v=1 / 2$. In a single tube $(N=1)$ at $v=1 / 2$ the checkerboard (CB)-type solid can exist only if the two-body repulsion exceeds a certain threshold. The situation becomes dramatically different in the cases $N>2$. As will be discussed below, the bosonization analysis shows that, even in the absence of any repulsion, the intertube attraction induces the 


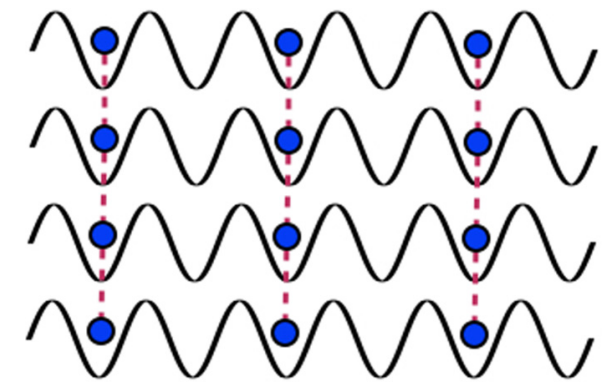

FIG. 5. (Color online) A sketch of the CB phase. The dashed lines indicate binding between atoms from different tubes at $v=1 / 2$.

CB insulator in the limit $V_{d} \rightarrow 0$ as long as $N \geqslant 3$. This conclusion is consistent with our ab initio simulations. A sketch of this phase is shown in Fig. 5.

Solids at other rational fillings $v=1 / 3,1 / 5,2 / 5, \ldots$ are possible as well. To induce them, however, the interaction $\sim V_{d}$ must exceed the corresponding thresholds determined by the denominators of the fractions. Concluding this section we note that in an insulating state the renormalized Luttinger matrix $\hat{K}$ in the action (12) is zero which implies no off-diagonal algebraic order.

\section{IV. $N$-TUBE BOSONIZATION}

Here we will discuss the phases described in Secs. III B, IIIC, IIID, and IIIE within the framework of the bosonization approach [34] in order to reveal their thresholdless nature. The bosonic field operator $\psi_{z}(x)$ is represented in terms of the superfluid phase $\phi_{z}$ and the density $\rho_{z}=\left|\psi_{z}\right|^{2}$, which can be expressed in terms of Haldane's "angle" variable $\theta_{z}[34]$ as

$$
\rho_{z}(x)=\left(v_{z}+\frac{1}{\pi} \nabla_{x} \theta_{z}\right) \sum_{m_{z}=0, \pm 1, \ldots} e^{\left[i 2 m_{z}\left(\theta_{z}+\pi v_{z} x\right)\right]},
$$

where $\theta_{z}(x)$ is conjugate to the superfluid phase $\phi_{z}$. The term $m_{z}=0$ gives the forward scattering (FS) interaction and the terms with $m_{z} \neq 0$ account for the back scattering (BS) events.

In the absence of intertube tunneling, the bosonized action corresponding to the Hamiltonian (1) is

$$
S=\sum_{z} S_{z}^{(0)}+\sum_{z, z^{\prime}}\left[S_{z, z^{\prime}}^{(\mathrm{FS})}+S_{z, z^{\prime}}^{(\mathrm{BS})}\right]
$$

where

$$
S_{z}^{(0)}=\int_{0}^{\beta} d \tau \int d x \frac{1}{2 \pi K}\left[\left(\nabla_{\tau} \theta_{z}(x, \tau)\right)^{2}+\left(\nabla_{x} \theta_{z}(x, \tau)\right)^{2}\right],
$$

in units $V_{s}=1$. Here $K$ is the bare Luttinger liquid parameter, that is, not yet renormalized by the interactions. For hard-core bosons and zero dipolar interaction, each tube is equivalent to a $X X Z$ spin $S=1 / 2$ chain with zero $S_{z}-S_{z}$ coupling. Accordingly, $K=1$ (see in Ref. [19]). In the following we use periodic boundary conditions along $x$ and $z$ coordinates.

The second term in Eq. (14) is the FS part of the action, $S_{z, z^{\prime}}^{(\mathrm{FS})}=\frac{1}{2 \pi^{2}} \int_{0}^{\beta} d \tau \int d x \int d x^{\prime} V\left(x-x^{\prime}, z-\right.$ $\left.z^{\prime}\right) \nabla_{x} \theta_{z}(x, \tau) \nabla_{x^{\prime}} \theta_{z^{\prime}}\left(x^{\prime}, \tau\right)$. In the long wave limit of the space-time Fourier representation $\left(q_{x} \rightarrow 0\right)$ the FS part of the action becomes

$$
S_{z, z^{\prime}}^{(\mathrm{FS})}=\frac{1}{2} \sum_{\vec{q}} \bar{V}\left(z-z^{\prime}\right) q_{x}^{2} \tilde{\theta}_{z}(\vec{q}) \tilde{\theta}_{z^{\prime}}(-\vec{q}) .
$$

Here, the summation is performed over the time-space harmonics, $\vec{q}=\left(\omega, q_{x}\right)$ along each tube and

$$
\bar{V}(z)=\frac{1}{\pi^{2}} \int d x V(x, z) .
$$

In particular, for the dipole-dipole interaction given by Eq. (2) one finds

$$
\bar{V}\left(z-z^{\prime}\right)=-\frac{\gamma_{1}}{\left(z-z^{\prime}\right)^{2}}, \quad \gamma_{1} \approx \frac{2.00 V_{d}}{\pi^{2}},
$$

for $\left|z-z^{\prime}\right|=1,2,3, \ldots$ For $z=z^{\prime}$, the dipolar interaction is purely repulsive, with $\bar{V}(0)=\int d x V(x, z=0)$, and it must be cut off at some short distance. Here we consider the same length scale along $z$ and $x$, and choose the cutoff at $x=1$ such that $\bar{V}(0) \approx 2.40 V_{d} / \pi^{2}$. Thus, in the long-wave limit, while the interlayer interaction is attractive, the intralayer one is repulsive. In the case of the purely repulsive dipolar interaction, Eq. (3), the FS interaction given by Eq. (18) changes sign, that is, $\gamma_{1} \rightarrow-\gamma_{1}$ for $z \neq z^{\prime}$ [with $\bar{V}(0)$ being unchanged].

Next, we introduce Fourier harmonics along the $z$ coordinate, $\tilde{\theta}_{q_{z}}(\vec{q})$ and $\tilde{V}\left(q_{z}\right)$, corresponding to $\tilde{\theta}_{z}(\vec{q})$ and $\bar{V}(z)$, respectively. We can now write the Gaussian part $\sum_{z} S_{z}^{(0)}+$ $\sum_{z, z^{\prime}} S_{z, z^{\prime}}^{(F S)}$ of the action (14) as

$$
S_{0}=\sum_{\vec{q}, q_{z}}\left[\frac{1}{2 \pi K} \vec{q}^{2}+\frac{1}{2} \tilde{V}\left(q_{z}\right) q_{x}^{2}\right]\left|\tilde{\theta}\left(\vec{q}, q_{z}\right)\right|^{2} .
$$

Equation (19) implies the renormalization of the speed of sound $V_{s} \rightarrow \tilde{V}_{s}\left(q_{z}\right)=\sqrt{1+\pi K \tilde{V}\left(q_{z}\right)}$ (in units of the bare value) as well as of the Luttinger parameter,

$$
K \rightarrow \tilde{K}\left(q_{z}\right)=\frac{K}{\sqrt{1+\pi K \tilde{V}\left(q_{z}\right)}} .
$$

Thus, both quantities $\tilde{V}_{s}\left(q_{z}\right), \tilde{K}\left(q_{z}\right)$ depend on the wave vector $q_{z}$ counting the layers so that the action (19) takes the form (11). As discussed above, in what follows we will ignore the renormalization of the speed of sound and will rather consider the form (12). Then, in terms of the dual variables $\theta_{z}$ the gradient part of the renormalized action becomes

$$
S_{R}=\int d x \int_{0}^{\beta} d \tau \sum_{z, z^{\prime}} \frac{1}{2 \pi}\left(\hat{K}^{-1}\right)_{z, z^{\prime}} \vec{\nabla} \theta_{z} \vec{\nabla} \theta_{z^{\prime}},
$$

where $\left(\hat{K}^{-1}\right)_{z, z^{\prime}}$ is the inverse of the renormalized Luttinger matrix $\hat{K}$ introduced in Eq. (12).

Finally, the third term in Eq. (14) accounts for the backscattering events [34] which in the context of the system studied can be written as

$$
\begin{aligned}
S_{z, z^{\prime}}^{(\mathrm{BS})}= & -\int d \tau \sum_{m_{z}, m_{z^{\prime}}} \sum_{x=0,1, \ldots L} V_{m_{z}, m_{z^{\prime}}}\left(z, z^{\prime}\right) \cos \left[2 \left(m_{z} \theta_{z}\right.\right. \\
& \left.\left.+m_{z^{\prime}} \theta_{z^{\prime}}\right)+2 \pi\left(v_{z} m_{z}+v_{z^{\prime}} m_{z^{\prime}}\right) x\right],
\end{aligned}
$$

where the amplitudes $V_{m_{z} ; m_{z^{\prime}}^{\prime}}\left(z, z^{\prime}\right)$ are induced by the interaction and satisfy the renormalization flow (to be derived in the 
standard one-loop approximation in Appendix A). While the FS sets in the initial value of the Luttinger matrix Eq. (20), the $\mathrm{BS}$ is responsible for its further renormalization.

The backscattering (22) is responsible for the formation of the phases discussed above. In order to make it effective, though, the filling factors must satisfy the corresponding matching conditions so that the term $\propto x$ under the cos in Eq. (22) remains relevant. Otherwise, it will be washed out by the integration over the tube length. As an example, in the composite superfluid phase of $N$ layers all superfluid phases $\phi_{z}$ are disordered except for the total $\sum_{z=1, N} \phi_{z}$. Accordingly, all the differences $\theta_{z}-\theta_{z^{\prime}}$ must be gapped (not fluctuating) as the variables conjugated to $\phi_{z}-\phi_{z^{\prime}}$. This is possible when the term $2 \pi\left(v_{z} m_{z}+v_{z^{\prime}} m_{z^{\prime}}\right) x$ in Eq. (22) is zero for $m_{z}=1, m_{z^{\prime}}=-1$, that is, $v_{z}=v$ for each $z$. Otherwise, the cos term can be neglected and the fluctuations of all $\theta_{z}$ (and thus $\phi_{z}$ ) will feature algebraic order, that is, $N$ atomic superfluids.

In the limit $V_{d} \rightarrow 0$ the RG flows can be found exactly. First we remind that for hard-core bosons $K_{z z}=1$ and $K_{z z^{\prime}}=0$ for $z \neq z^{\prime}$ at $V_{d}=0$. Therefore, as it will be clear below, in the limit of small interactions, only the lowest nontrivial values of $m_{z}, m_{z^{\prime}}\left(m_{z}= \pm 1, m_{z^{\prime}}= \pm 1\right)$ can become relevant in the sum (22), provided $v_{z} m_{z}+v_{z^{\prime}} m_{z^{\prime}}=0, \pm 1$. Hence, the relevance of the backscattering for a particular pair $z, z^{\prime}$ of layers can be controlled by adjusting the bosonic populations in individual tubes.

\section{A. RG for the composite superfluid}

Due to the spatially nonlocal nature of dipolar interactions, the composite superfluid phase, Sec. III C, can form between tubes with the same filling factors regardless of their geometrical positions. For example, in a system of $N=6$ tubes where $v_{1}=v_{2}=v_{5}=v$ (here we consider $v \neq$ $1 / 2)$, with all other values $v_{3} \neq v_{4} \neq v_{6} \neq v$, the harmonics $V_{1 ;-1}(1,2), V_{1 ;-1}(1,5), V_{1 ;-1}(2,5)$ can become relevant, while all others remain irrelevant [simply because of the oscillating phases $2 \pi\left(v_{z} m_{z}+v_{z^{\prime}} m_{z^{\prime}}\right) x$, with $z, z^{\prime}=3,4,6$, in the corresponding cos-harmonics in Eq. (22)].

The RG equations for the amplitudes of the corresponding harmonics $\left(z \neq z^{\prime}\right)$ between the tubes with identical filling factors are (see Appendix A)

$$
\frac{d V_{1 ;-1}\left(z, z^{\prime}\right)}{d \ln l}=\left[2-K_{z z}-K_{z^{\prime} z^{\prime}}+2 K_{z z^{\prime}}\right] V_{1 ;-1}\left(z, z^{\prime}\right),
$$

where $K_{z z^{\prime}}$ are the matrix elements of the matrix $\hat{K}$ in Eq. (12) and the initial (bare) values of the amplitudes $V_{1 ;-1}\left(z, z^{\prime}\right)$ are determined by the dipolar interactions. In the limit of no interactions, that is, $\bar{V} \rightarrow 0$, the RG flow starts from the critical point determined by the factor $\left[2-K_{z z}-K_{z^{\prime} z^{\prime}}+2 K_{z z^{\prime}}\right] \rightarrow 0$ (since $K_{z z^{\prime}} \rightarrow K \delta_{z z^{\prime}}$, with $K=1$ ) in Eq. (23). As explained in Appendix A, Eq. (A3), this factor is $\propto V_{d}$ and is positive for the case of the attractive interlayer interaction (18). Thus, the relevant amplitudes diverge as $V_{1 ;-1}\left(z, z^{\prime}\right) \sim l^{b}$ with $b \sim V_{d}$. While formally this implies that CSF is induced by an arbitrary small interlayer attraction $V_{d}$, a physical scale $l_{\mathrm{CSF}}$ on which such a composite phase can be observed is actually exponentially divergent as $l_{\mathrm{CSF}} \sim \exp \left(\ldots 1 / V_{d}\right) \rightarrow \infty$, where “..." means a coefficient $\sim 1$ (see below).
Using the $N=6$ example from above, the formation of the CSF between the tubes $z=1,2,5$ implies that the harmonics $V_{1 ;-1}(1,2), V_{1 ;-1}(1,5), V_{1 ;-1}(2,5)$ exhibit the runaway flow to $\infty$ in Eq. (23), while all other combinations can be set essentially to zero. In other words, two $U(1)$ symmetries are being restored so that the system is described by the algebraic orders in $\psi_{3}, \psi_{4}, \psi_{6}$ and in the CSF field $\Psi_{1,2,5}=\psi_{1} \psi_{2} \psi_{5}$.

In the case of translational invariance along the $z$ axis, that is, when $v_{z}=v$ for all tubes, Eq. (23) can be explicitly written in terms of the kernels of the Luttinger matrix and its inverse as

$$
\frac{d V_{1 ;-1}(z)}{d \ln l}=2[1-K(0)+K(z)] V_{1 ;-1}(z),
$$

where we have taken into account that the amplitudes $V_{1 ;-1}\left(z, z^{\prime}\right)$ as well as the matrix elements $K_{z z^{\prime}}$ are functions of the difference $z-z^{\prime}$ rather than of $z, z^{\prime}$ separately: $V_{1 ;-1}\left(z, z^{\prime}\right) \equiv V_{1 ;-1}\left(z-z^{\prime}\right),(\hat{K})_{z z^{\prime}} \equiv K\left(z-z^{\prime}\right),\left(\hat{K}^{-1}\right)_{z z^{\prime}} \equiv$ $K^{-1}\left(z-z^{\prime}\right)$, where

$$
\begin{aligned}
K(z) & =\frac{1}{N} \sum_{q_{z}} \tilde{K}\left(q_{z}\right) \mathrm{e}^{i q_{z} z}, \\
K^{-1}(z) & =\frac{1}{N} \sum_{q_{z}} \frac{1}{\tilde{K}\left(q_{z}\right)} \mathrm{e}^{i q_{z} z},
\end{aligned}
$$

with the corresponding inverse transformations.

Ignoring the renormalization of the Luttinger matrix by the BS, the value of $\tilde{K}\left(q_{z}\right)$ from Eq. (20) can be used in Eq. (24) in the limit $V_{d} \rightarrow 0$. Then, in the lowest order in $V_{d}$ we find

$$
\frac{d V_{1 ;-1}(z)}{d \ln l} \approx \pi(\bar{V}(0)-\bar{V}(z)) V_{1 ;-1}(z),
$$

where $\bar{V}(z)$ is given in Eq. (18) and $K$ is set to its value, $K=1$, for noninteracting tubes. Thus, for $\bar{V}(0)>0$ and $\bar{V}\left(z-z^{\prime}\right)<0$, as it is in the case of the dipolar interaction between molecules polarized along the $z$ axis, Eq. (18), the harmonics $V_{1 ;-1}\left(z-z^{\prime}\right) \cos \left(2 \theta_{z}-2 \theta_{z^{\prime}}\right)$ become relevant for arbitrary small $V_{d}$. This implies that the superflow is only possible in the channel of the center-of-mass motion of all tubes because relative density fluctuations are gapped. It is also interesting to note that, in the case of the purely repulsive interaction (3) (that is, when the molecules are polarized along the $y$ axis), where $\bar{V}(z)>0$ for $|z|>0$, the composite superfluid, CSF, is also possible as long as $\bar{V}(0)>\bar{V}(z)>0$. This binding caused by repulsion is a specific property of $1 \mathrm{D}$ geometry.

The renormalization of the BS amplitudes, Eq. (24), should be considered together with the renormalization of the matrix $\hat{K}$ in Eq. (12). As explained in Appendix A, these equations are

$$
\frac{d\left(\hat{K}^{-1}\right)_{z z^{\prime}}}{d \ln l}=-C\left[V_{1,-1}\left(z, z^{\prime}\right)\right]^{2}\left(K_{z z}+K_{z^{\prime} z^{\prime}}-2 K_{z z^{\prime}}\right),
$$

for the off-diagonal terms, $z \neq z^{\prime}$, and

$$
\frac{d\left(\hat{K}^{-1}\right)_{z z}}{d \ln l}=C \sum_{z^{\prime}}\left[V_{1,-1}\left(z, z^{\prime}\right)\right]^{2}\left(K_{z z}+K_{z^{\prime} z^{\prime}}-2 K_{z z^{\prime}}\right),
$$

for the diagonal ones. Here the constant $C>0$ depends on the type of the short-distance cutoff (see in Ref. [19]). This 
constant can be absorbed into the definition of $V_{1,-1}(z)$ by simple rescaling of the amplitudes. It is worth noting that only the pairs $\left(z, z^{\prime}\right)$ such that $v_{z}=v_{z^{\prime}}$ are involved in Eqs. (28) and (29).

In the case of the translational invariance, that is, $v_{z}=v$, these equations become

$$
\frac{d K^{-1}(z)}{d \ln l}=-2 C\left[V_{1,-1}(z)\right]^{2}(K(0)-K(z)),
$$

where $z \neq 0$ and

$$
\frac{d K^{-1}(0)}{d \ln l}=\sum_{z} 2 C\left[V_{1,-1}(z)\right]^{2}(K(0)-K(z)),
$$

and they should be considered self-consistently together with Eqs. (24)-(26). An elementary inspection of Eqs. (24)(26), (30), and (31) shows that $\tilde{K}\left(q_{z}=0\right)$ is not affected by the RG because $\sum_{z} \frac{d K^{-1}(z)}{d \ln l}=0$. This implies that the field $\Psi_{\mathrm{CSF}}=$ $\psi_{1} \psi_{2} \ldots \psi_{N}$ always remains condensed. Furthermore, as long as the initial flow of $V_{1 ;-1}(z)$ [described by Eq. (27)] drives the amplitudes away to $\infty$, the fixed point for the Luttinger matrix is given by $K(z)=K(0)$, that is, by $\tilde{K}\left(q_{z} \neq 0\right)=0$.

\section{B. RG for supercounterfluids}

If there is a pair of tubes $z, z^{\prime}\left(z \neq z^{\prime}\right)$ with filling factors $v_{z} \neq 1 / 2$ and $v_{z^{\prime}}=1-v_{z}$, the BS harmonic $V_{1 ; 1}\left(z, z^{\prime}\right) \cos \left(2 \theta_{z}+2 \theta_{z^{\prime}}\right)$ can become relevant, while $V_{1 ;-1}\left(z, z^{\prime}\right)$ is irrelevant due to the mismatch of the filling factors. As a consequence, the gapless superflow is possible only in the counterflow channel. In other words, it is the difference between the two phases which remains gapless.

The RG equations for the counterflow can be written for each pair $z, z^{\prime}$ of tubes with the complementary filling factors by simply changing the sign in front of the $K_{z z^{\prime}}$ term in the corresponding equations, Eq. (23), derived above for the complex superfluids (see details in Appendix A). Specifically, we find

$$
\frac{d V_{1 ; 1}\left(z, z^{\prime}\right)}{d \ln l}=\left[2-K_{z z}-K_{z^{\prime} z^{\prime}}-2 K_{z z^{\prime}}\right] V_{1 ; 1}\left(z, z^{\prime}\right) .
$$

Here, in full analogy with the composite superfluids, the $V_{1 ; 1}$ channel can become gapped in the limit $V_{d} \rightarrow 0$.

For the case of more than two tubes in the counterflow regime, the dipolar interaction can induce an additional gap in the $\sim V_{1 ;-1}$ channel in tubes with identical filling factors. However, a simple count of the remaining gapless phases shows that the gap in the $V_{1 ;-1}$ channel does not change their number. Indeed, let's consider two sets of tubes, $M_{1}>1$ and $M_{2}>1$, so that in the first one the filling factor in each tube is $v \neq 1 / 2$ and in the second one it is $1-v$. Then, there will be gaps in the channels $V_{1 ;-1}\left(z_{1}, z_{1}^{\prime}\right)$ for each pair $z_{1}, z_{1}^{\prime}$ from the first set of $M_{1}$ tubes and in $V_{1 ;-1}\left(z_{2}, z_{2}^{\prime}\right)$ for each pair $z_{2}, z_{2}^{\prime}$ from the other set. As a result, there are two total phases from each group left gapless. Then, the channels $V_{1 ; 1}\left(z_{1}, z_{2}\right)$ also become gapped due to the counterflow BS. This leaves just one phase gapless. The described situation has a very simple interpretation: the gaps in tubes with equal filling factors imply formation of a pair of composite superfluids - one per each group of tubes and these composite superfluids further bind in the counterflow regime, as discussed in the Sec. III D.
Similarly to the composite superfluids, Eq. (28), the Luttinger matrix satisfies (see Appendix A)

$$
\frac{d\left(\hat{K}^{-1}\right)_{z z^{\prime}}}{d \ln l}=C\left[V_{1 ; 1}\left(z, z^{\prime}\right)\right]^{2}\left(K_{z z}+K_{z^{\prime} z^{\prime}}+2 K_{z z^{\prime}}\right),
$$

where $z \neq z^{\prime}$, and

$$
\begin{aligned}
\frac{d\left(\hat{K}^{-1}\right)_{z z}}{d \ln l}= & C \sum_{z^{\prime} \neq z}\left\{\left[V_{1 ; 1}\left(z, z^{\prime}\right)\right]^{2}\left(K_{z z}+K_{z^{\prime} z^{\prime}}+2 K_{z z^{\prime}}\right)\right. \\
& \left.+\left[V_{1 ;-1}\left(z, z^{\prime}\right)\right]^{2}\left(K_{z z}+K_{z^{\prime} z^{\prime}}-2 K_{z z^{\prime}}\right)\right\}
\end{aligned}
$$

The first sum here is the contribution from the pairs of tubes with complementary filling factors, and the second one is due to the tubes with the same filling factors.

Finally, we write the above equations for the case of translational symmetry along the $z$ axis. This can be realized when, for example, tubes with even $z$ coordinates $(z=0,2,4, \ldots)$ have filling factor $v$ and tubes with odd $z(z=1,3,5, \ldots)$ have filling factor $1-v$ (see Fig. 4). Then, similarly to the composite superfluid case,

$$
\frac{d V_{1 ; 1}(z)}{d \ln l}=2[1-K(0)-K(z)] V_{1 ; 1}(z),
$$

where the distance $z=z-z^{\prime}=1,3,5, \ldots$ corresponds to pairs of tubes with the complementary filling factors. For the distances $z=2,4,6, \ldots$, that is, for layers with same filling factors, Eq. (24) has to be used. Similarly the flow of the matrix of stiffnesses at odd distances $z$ is given by

$$
\frac{d K^{-1}(z)}{d \ln l}=2 C\left[V_{1 ; 1}(z)\right]^{2}(K(0)+K(z)),
$$

while even, nonzero distances $z$ are described by Eq. (30), and the diagonal term has contribution from all the pairs of tubes,

$$
\begin{aligned}
\frac{d K^{-1}(0)}{d \ln l}= & 2 C \sum_{z=1,3,5, \ldots}\left[V_{1 ; 1}(z)\right]^{2}(K(0)+K(z)) \\
& +2 C \sum_{z=2,4,6, \ldots}\left[V_{1 ;-1}(z)\right]^{2}(K(0)-K(z)) .
\end{aligned}
$$

It is instructive to ignore Eqs. (36) and (37), and substitute the initial value (20) into Eq. (35) in the limit $V_{d} \rightarrow 0$. For $z \neq 0$ this gives

$$
\frac{d V_{1 ; 1}(z)}{d \ln l} \approx \pi(\bar{V}(0)+\bar{V}(z)) V_{1 ; 1}(z),
$$

where we have only used the first-order term in $V_{d} \rightarrow 0$ while expanding (20). Thus, for the purely repulsive interaction, Eq. (3), the harmonic $V_{1 ; 1}(z)$ is relevant for arbitrary small $V_{d}$ in a direct analogy with the CSF case. Furthermore, it is interesting to note that interlayer attraction $\bar{V}(z)<0$, Eq. (18), also induces the composite supercounterfluid as long as the intralayer repulsion is strong enough, that is, $\bar{V}(0)>|\bar{V}(z)|$.

The analysis of the above equations shows that for even number of layers, the fixed point corresponds to $K(z)=$ $K(0)$ for $z=2,4,6, \ldots$ and $K(z)=-K(0)$ for $z=1,3,5, \ldots$ Thus, while $\tilde{K}\left(q_{z}=0\right)=0$, the harmonic at $q_{z}=\pi$ remains condensed [because $\tilde{K}\left(q_{z}=\pi\right) \neq 0$ ]. This, as discussed earlier, corresponds to the supercounterfluidity in the nearest neighbor layers, with the condensed field $\Psi_{\mathrm{SCF}}=$ $\psi_{1} \psi_{2}^{\dagger} \psi_{3} \psi_{4}^{\dagger} \ldots$ 


\section{RG for $v=1 / 2$ insulators}

In the absence of intertube interactions, hard-core bosons can form a checkerboard (CB) insulator at filling factor $v=1 / 2$ only if the repulsive interaction is strong enough, so that the Luttinger parameter $K$ is reduced from $K=1$ to $K=1 / 2$ (see Ref. [19]). This can also be seen from Eq. (32) written for $z=z^{\prime}$, that is, for the intratube harmonic $\cos \left(4 \theta_{z}+\right.$ $\left.4 \pi v_{z} x\right)$. In this case, Eq. (32) becomes $d V_{1 ; 1}(0) / d \ln l=$ $\left(2-4 K_{z z}\right) V_{1 ; 1}(0)$. In the absence of intertube interaction the Luttinger matrix becomes diagonal $(\hat{K})_{z z^{\prime}}=K \delta_{z z^{\prime}}$, so that one can write $d V_{1 ; 1}(0) / d \ln l=(2-4 K) V_{1 ; 1}(0)$, implying that the critical value $K=K_{c}=1 / 2$.

The situation changes dramatically in the presence of intertube interaction. At filling $v=1 / 2$ harmonics $V_{1 ; 1}(z), V_{1 ;-1}(z)$ with $z \neq 0$ can become relevant for $K=1$, as Eqs. (27) and (38) indicate. This happens regardless of the sign of the intertube interaction even in the limit $V_{d} \rightarrow 0$ as long as $\hat{V}(0)>|\hat{V}(z)|$. Accordingly, all pairs of phases $\theta_{z} \pm \theta_{z^{\prime}}$, with $z \neq z^{\prime}$ become gapped, which implies that all the individual phases $\theta_{z}$ are gapped.

It is possible to make a much stronger statement: For $N>2$, the $v=1 / 2$ insulating state occurs even in the absence of intratube interaction, i.e., $\bar{V}(0)=0$, and for purely attractive intertube interaction $\bar{V}(z) \rightarrow 0$. In order to see this, we analyze the RG Eq. (24) which, as the initial flow (27) indicates, implies relevance of all $V_{1 ;-1}(z)$ for $z \neq 0$. Accordingly, as Eqs. (30) and (31) show, the matrix $\hat{K}$ flows toward $\tilde{K}\left(q_{z}\right)=0$ (in the limit $\ln l \rightarrow \infty$ ) for all $q_{z}$ except $q_{z}=0$. This means Eq. (35) can be approximately rewritten as

$$
\frac{d V_{1 ; 1}(z)}{d \ln l} \approx\left[2-\frac{4}{N} \tilde{K}(0)\right] V_{1 ; 1}(z),
$$

in the limit $\ln l \rightarrow \infty$. Keeping in mind that at small $V_{d} \rightarrow 0$ the initial value $\tilde{K}(0) \approx 1$, this equation shows that, even if the renormalization of $\tilde{K}(0)$ is ignored, $V_{1 ; 1}(z)$ flows to $\infty$ as $\sim l^{2-4 / N}$ as long as $N>2$ [which means that the harmonic $q_{z}=0$ is also gapped and $\tilde{K}(0)$ must actually flow to 0$]$. As it will be seen below, this conclusion is also consistent with the simulations.

The $N=2$ case is special because, in the one-loop approximation, the RG equations for $V_{1 ;-1}$ and $\tilde{K}(\pi)$ are independent from the equations for $V_{1 ; 1}$ and $\tilde{K}(0)$. Accordingly, the equation for $\tilde{K}(0)$ predicts that it must flow to a stable fixed point $\tilde{K}(0)>1$ as long as $\bar{V}(0)<|\bar{V}(z)|$ and $\bar{V}(z)<0$. This issue will be discussed in greater detail elsewhere. Below we will explicitly demonstrate numerically the thresholdless nature of the composite superfluid in the simplest case $N=2$.

\section{QUANTUM MONTE CARLO AB-INITIO ALGORITHM AND SOME RESULTS}

The standard worm algorithm (WA) [31] is based on the evaluation of one-particle correlators $D_{1}$ in imaginary time and the possibility of switching effectively to the functional space of the partition function of the closed world lines of particles. If $M=2,3, \ldots$, particles (or holes) form bound complexes, the efficient simulations can be achieved only through evaluation of the $M$-particle correlators. In this case $M=2$, such an algorithm has been developed in Ref. [5].
The situation becomes more complicated for $M>2$, when no effective switching to the partition function space can, in general, be achieved. This problem has been resolved in Ref. [30] in the case of no interlayer tunneling and in Ref. [22] in a more general setting. Here the algorithm [30] (designed to work in a discrete space time) is extended to the quantum case, that is, to continuous time.

While avoiding technical details, here we give a general overview of the quantities measured during the simulations. The most general correlator which can effectively describe a phase of $M$ bound complexes is the $M$-particle correlator-a function of $6 M$ variables,

$D_{M}\left(r_{1}, \ldots, r_{M} ; r_{1}^{\prime}, \ldots, r_{N}^{\prime}\right)=\left\langle A^{\dagger}\left(r_{1}, \ldots, r_{M}\right) A\left(r_{1}^{\prime}, \ldots, r_{M}^{\prime}\right)\right\rangle$,

where $\langle\ldots\rangle$ stands for quantum-statistical averaging with the weight $\exp (-\beta H)$ determined by the Hamiltonian $H$ (1) and $A\left(r_{1}, \ldots, r_{M}\right)=a\left(r_{1}\right) a\left(r_{2}\right) \ldots a\left(r_{M}\right)$, with $a\left(r_{i}\right)$ being the bosonic annihilation operator in the space-time point $r_{i}=$ $\left(x_{i}, z_{i}, \tau_{i}\right)$, with $i=1,2, \ldots, M$. The imaginary time dependence is given by the interaction representation defined for an operator $f$ as $f(\tau)=e^{\tau H_{0}} f(0) e^{-\tau H_{0}}$, where $f(0)$ is the operator in the Schrödinger representation and $H_{0}$ is the part of the Hamiltonian which is diagonal in the Fock basis, that is, the interaction part of $H$.

Evaluation of $D_{M}$ is based on the random walks of the $2 M$ open ends of the world lines, worms [31], controlled by the famous Metropolis prescription. The identification of the phases, then, stems from the statistics of the relative distances between the worms, as described in Ref. [30]. For example, in the CSF phase of complexes each composed of $M$ particles, all the correlators $D_{M^{\prime}}$ (40) with $M^{\prime}<M$ exhibit exponential decay with respect to all the pairs of space-time distances $r_{i}-r_{j}, r_{i}^{\prime}-r_{j}^{\prime}$, and $r_{i}-r_{j}^{\prime}$ where $i, j=1,2, \ldots, M^{\prime}$. This behavior is the key signature of insulators with no off-diagonal long-range (or algebraic) order. A completely different behavior is demonstrated by the correlator $D_{M}$. On the one hand, if all the ends from one set, e.g., $r_{1}, r_{2}, \ldots, r_{M}$ are kept inside a small region, the ends from the other set will automatically stay close together within some finite radius $\xi_{0}$ determining a typical extension of the constituents forming one complex, that is, $\left\langle\left|r_{i}^{\prime}-r_{j}^{\prime}\right|\right\rangle \leqslant \xi_{0}$. On the other hand, the dependence of $D_{M}$ on the relative space-time distance between the "centers of mass" $\left|R_{\text {c.m. }}-R_{\text {c.m. }}^{\prime}\right|$ (defined as $R_{\text {c.m. }}=\left[r_{1}+\cdots+r_{M}\right] / M$ and $\left.R_{\text {c.m. }}^{\prime}=\left[r_{1}^{\prime}+\cdots+r_{M}^{\prime}\right] / M\right)$ features the off-diagonal long-range (or algebraic) order. The transition from CSF to the standard superfluid (where $D_{1}$ is long ranged) is marked by the divergence of $\xi_{0}$.

Keeping in mind the specificity of the present system, we evaluated the correlator $D_{N}$ and kept only one pair of the variables $r_{i}, r_{i}^{\prime}$, one from the first set and one from the other, in each tube (there is no intertube tunneling so that each worm stays in its tube). In order to realize the "confinement" of the first set of variables $r_{1}, \ldots r_{N}$, we have introduced an artificial configuration weight $W \sim \exp \left[-\sum_{m, n}^{N}\left(\mid x_{m}-\right.\right.$ $\left.\left.x_{n}\left|/ \xi_{x}+\right| \tau_{m}-\tau_{n} \mid / \xi_{\tau}\right)\right]$, where $\xi_{x}$, $\xi_{\tau}$ are microscopic parameters chosen so as to maximize the algorithm efficiency. Accordingly, the expectation values $\langle\ldots\rangle$ are evaluated with respect to the weight $W \exp (-\beta H)$.

As demonstrated in Refs. [22,30], the described approach turned out to be very effective in identifying various phases 
as well as the universalities of the transitions. It can be easily adjusted to various systems. For example, if considering the bilayer system proposed in Ref. [20] the correlator $D_{p+q}$ should be used with $p$ pairs of the ends kept in one tube and $q$ pairs-in the other.

Here we present results of $a b$ initio quantum Monte Carlo (QMC) simulations based on the path integral with $H$ from Eq. (1) and focusing on demonstrating explicitly the absence of the threshold for the formation of the CSF state. Unless otherwise noted the simulations were performed in the case of nearest neighbor interlayer attraction, and in the absence of intralayer interactions. Specifically, we considered the case $N=2$ and compared the result for the renormalized Luttinger parameter determined numerically [through the representations $(6,7,10)]$ with the prediction of $\mathrm{RG}$. We have also performed simulations of the $N=3$ case within the approach described above and have demonstrated the following: (1) the formation of the CSF phase; (2) the existence of the insulating CB state of the chains at the filling $v=1 / 2$ and provided data consistent with the absence of the threshold for its formation.

\section{A. QMC study of the bilayer system, $N=2$ case}

Absence of the threshold for the phases discussed above implies that, in order to realize them, there is no need to pursue strong dipole-dipole interactions. Instead, the size of the system should be made large enough (and temperature low enough) so that the effects of small gaps are seen. Here we will address the issue of no threshold in detail by ab initio simulations of the bilayer system. The goal of this study is to demonstrate this property explicitly.

We consider two identical layers located at $z=0,1$ with $v_{0}=v_{1}=v$. We restrict the interparticle interactions to nearest neighbor interlayer attraction, where only particles directly on top of each other interact. The intralayer interactions are set to zero. Then, the Luttinger matrix consists of just two elements $\left(\hat{K}^{-1}\right)_{00}=\left(\hat{K}^{-1}\right)_{11}$ and $\left(\hat{K}^{-1}\right)_{01}$. Accordingly, the Fourier representation along the $z$ axis has just two harmonics with $q_{z}=0, \pi$, so that Eq. (26) yields $1 / \tilde{K}(0)=\left(\hat{K}^{-1}\right)_{00}+$ $\left(\hat{K}^{-1}\right)_{01}$ and $1 / \tilde{K}(\pi)=\left(\hat{K}^{-1}\right)_{00}-\left(\hat{K}^{-1}\right)_{01}$. As presented in Eqs. (8)-(10)

$$
\begin{aligned}
& \tilde{K}(\pi)=\frac{\pi}{2} \sqrt{\left\langle\left(W_{x}(0)-W_{x}(1)\right)^{2}\right\rangle\left\langle\left(W_{\tau}(0)-W_{\tau}(1)\right)^{2}\right\rangle}, \\
& \tilde{K}(0)=\frac{\pi}{2} \sqrt{\left\langle\left(W_{x}(0)+W_{x}(1)\right)^{2}\right\rangle\left\langle\left(W_{\tau}(0)+W_{\tau}(1)\right)^{2}\right\rangle},
\end{aligned}
$$

in terms of space-time windings $W_{x}(0), W_{x}(1), W_{\tau}(0), W_{\tau}(1)$.

We have determined $\tilde{K}(\pi)$ by QMC for various interactions and system sizes, where the RG scale $l$ was identified with the system size $L$, provided the inverse temperature $\beta=1 / T \propto L$ in the atomic units. Practically, we have kept $L \propto \beta$ so that $\left\langle\left(W_{x}(0)-W_{x}(1)\right)^{2}\right\rangle=\left\langle\left(W_{\tau}(0)-W_{\tau}(1)\right)^{2}\right\rangle$, in order to ensure space-time symmetry, that is, that the system is in its ground state.

Our purpose is comparing the numerical dependencies of $\tilde{K}(\pi)$ vs $L$ for various interaction strengths $V_{d}$ with the RG flows. The raw data for $\tilde{K}(\pi)$ are presented in Fig. 6. As it turned out, within the statistical errors of the simulations, the curves of $\tilde{K}(\pi)$ vs $L$ for various $0<V_{d} / J<1$ have been

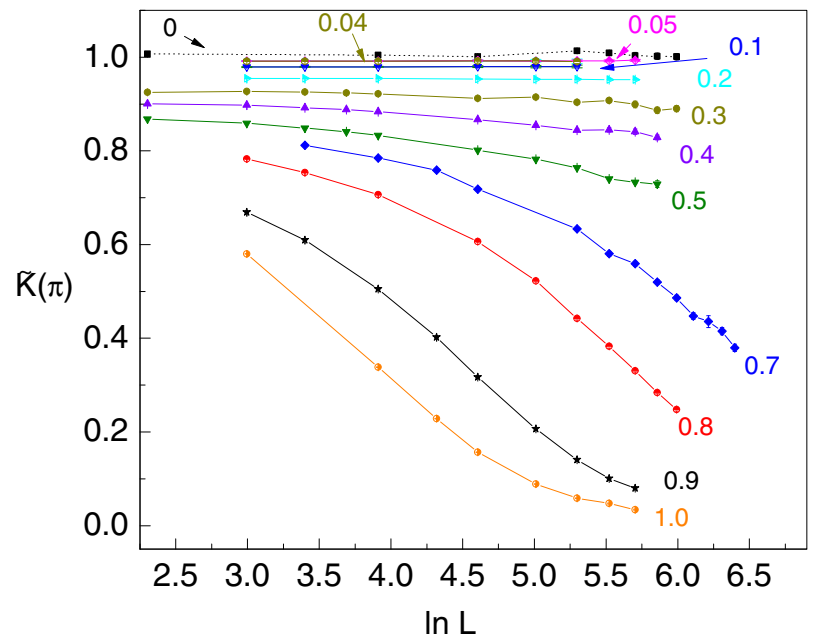

FIG. 6. (Color online) Numerical data for $\tilde{K}(\pi)$ as a function of system size $L$ for different values of the interlayer interaction $V_{d} / J$, and in the absence of intralayer repulsion.

found to belong to one master curve - the separatrix of the RG equations [Eqs. (B2)-(B4)] (discussed in Appendix B), which can be represented as

$$
\ln \xi_{s}-\frac{1}{\xi_{s}}=2 \ln \left(\frac{L}{L_{0}}\right), \quad \xi_{s}=\frac{1}{\tilde{K}(\pi)}-1,
$$

where $L_{0}\left(V_{d} / J\right)$ is a rescaling parameter which can be interpreted as the length $\xi_{0}$ - the size of a bound dimer. This dependence has been found from rescaling $\ln L \rightarrow$ $\ln L-\ln \left[L_{0}\left(V_{d}\right)\right]$ for each value of the interaction. The result of this procedure is presented in Fig. 7. As can be seen from

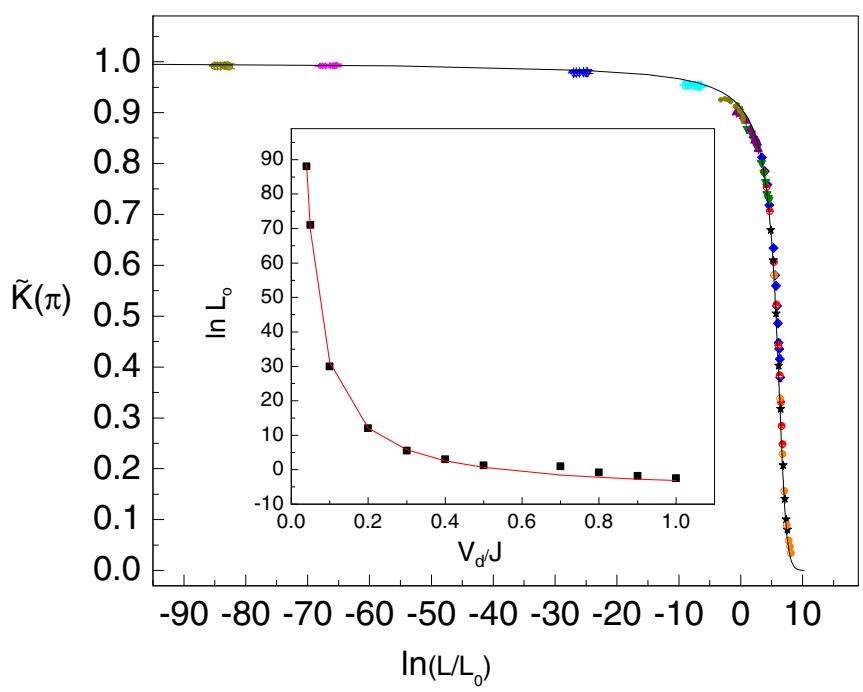

FIG. 7. (Color online) The data from Fig. 6 is shown vs $\ln \left(L / L_{0}\left(V_{d}\right)\right)$ with $L_{0}\left(V_{d}\right)$ chosen in such a way as to achieve the collapse on a single curve. The size of the symbols is determined by the statistical error bars. The solid line is the RG solution for the separatrix with the critical value of the Luttinger parameter being $K_{c}=1$, Eq. (43). (Inset) Plot of the rescaling parameter $\ln L_{0}$ vs $V_{d} / J$. Solid line is the fit by $\ln L_{0}=a /\left(V_{d} / J\right)-b$, with $a=3.82, b=6.96$. 
the inset, $L_{0}$ as a function of the intertube interaction diverges as

$$
L_{0} \sim \exp \left(\frac{a J}{\left|V_{d}\right|}\right), \quad V_{d} \rightarrow 0,
$$

where $a$ is a constant $(a=3.82)$. Such a divergence proves that the critical value for the formation of the dimer superfluid is $V_{d}=0$. Thus, the accurate matching of the numerical data by the RG solution (43) over almost 50 orders of magnitude of the (effective) distances as well as the dependence (44) indicate that paired superfluid is formed for infinitesimally small interlayer interaction strength. Such an approach-matching the numerical solution with the RG flow for finding the critical point of the Berezinskii-Kosterlitz-Thouless transition [35] has been pioneered in Ref. [36].

\section{B. QMC results for $\boldsymbol{N}=\mathbf{3}$ tubes}

Below we present QMC results obtained by the multiworm algorithm for the case of $N=3$ tubes. As has already been mentioned, achieving efficient numerical convergence by the approach [5] in the cases $N>2$ is not possible. Instead, the simulations should focus on evaluating the $N$-body correlator $D_{N}$ [Eq. (40)]. Then, the determination of the phases can be based on observing spatial dependencies of the corresponding correlators.

We introduce two quantities $f_{1}\left(x_{1}^{\prime}-x_{2}^{\prime}\right)$ and $f_{2}\left(x_{1}-x_{1}^{\prime}\right)$ which can be viewed as spatial projections of the full correlator $D_{3}$ where the pair $x_{1}^{\prime}, x_{2}^{\prime}$ in $f_{1}$ belongs to the "primed" coordinates in the definition (40), and $x_{1}, x_{1}^{\prime}$ in $f_{2}$ are from the "unprimed" and the "primed" sets, respectively. Specifically, $f_{1}\left(x_{1}^{\prime}, x_{2}^{\prime}\right) \propto \int d \tau_{1}^{\prime} d \tau_{2}^{\prime} d r_{1} d r_{2} d r_{3} d r_{3}^{\prime} D_{3} W$ and $f_{2}\left(x_{1}, x_{1}^{\prime}\right) \propto \int d \tau_{1} d \tau_{1}^{\prime} d r_{2} d r_{3} d r_{2}^{\prime} d r_{3}^{\prime} D_{3} W$, with the artificial weight $W$ discussed at the beginning of the Sec. V.

Given the definition, $f_{1}(x)$ must exhibit exponential decay in the $N=3$ CSF phase as well as in the insulating phases. The function $f_{2}$, while demonstrating the exponential decay in the insulator, should show algebraic behavior in the CSF phase. These features are clearly seen in Fig. 8 for $N=3$ identical tubes for two filling factors and in the presence of the dipolar interaction truncated to the third nearest neighbor at $V_{d} / J=0.75$. While the main plot clearly shows the CSF $(v=0.29)$, the inset represents the CB insulator $(v=1 / 2)$.

The CB phase is characterized by finite amplitude of the modulation of the density $\rho(x)=\sum_{z} n_{z}(x)$ at the wave vector $\pi$. The RG analysis conducted in Sec. IV C indicates that such modulation can occur even in the absence of the intralayer repulsion due to arbitrary small interlayer attraction $V_{d}$. In other words, the repulsive interaction causing the $\mathrm{CB}$ order is to be induced dynamically even if it is not present microscopically. This analysis, however, does not predict strength of such interaction. Using QMC simulations we studied the CB modulation in a system where the interparticle interaction is restricted to nearest neighbor interlayer attraction. We were able to resolve the CB modulation only for $V_{d} / J \geqslant 0.38$. Furthermore, the structure factor correlator $S(x)=(-1)^{x}\langle\rho(x) \rho(0)\rangle$ showed a very weak dependence on the system size $L$ for the whole range of measurements $0.38 \leqslant V_{d} / J \leqslant 1$. In these circumstances conducting the comparison with the RG flow like it was done for the case of the paired superfluid does not appear to be

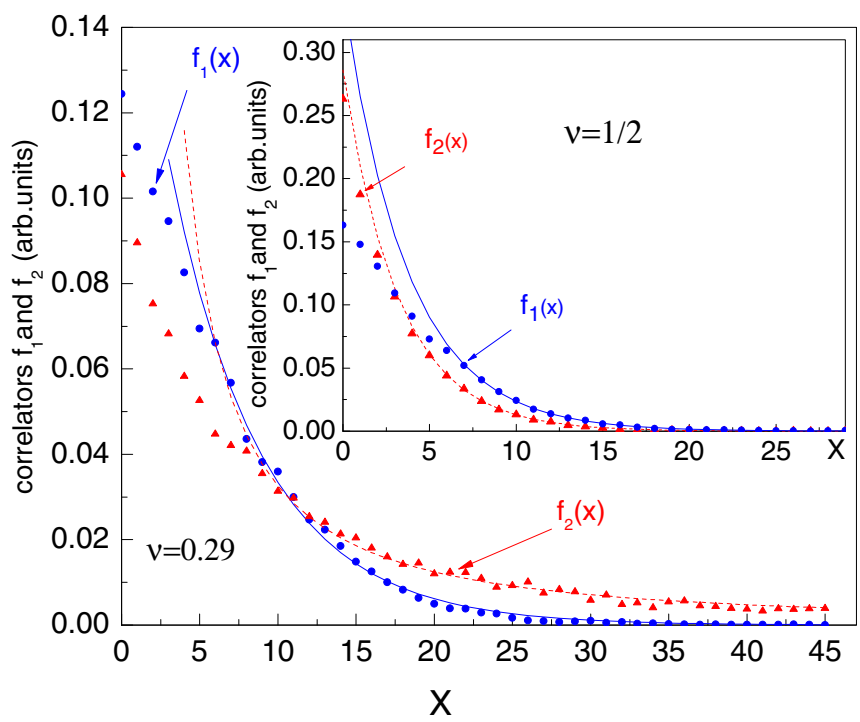

FIG. 8. (Color online) The CSF and CB phases in the $N=3$ tubes. QMC data (points) for the correlators $f_{1}(x)$ and $f_{2}(x)$ and their fits (lines) in the presence of dipolar interaction. The filling factors are shown. (Main panel) While $f_{1}(x)$ exhibits the exponential decay $\sim \exp (-0.169|x|), f_{2}(x)$ shows algebraic order $\propto|x|^{-1.39}$. (Inset) In the CB phase both functions are exponentially decaying, $f_{1}(x) \sim$ $\exp (-0.269|x|), f_{2}(x) \sim \exp (-0.310|x|)$.

feasible. In other words, a weak dependence of the induced repulsion on $V_{d}$ does not allow approaching the critical region at small $V_{d}$. Thus, the observed CB order corresponds to the values of the renormalized Luttinger parameter which are already so small that the structure factor $\sim C_{2}|x|^{-2 K}$ becomes essentially independent of $x$, with the factor $C_{2}$ being a nonuniversal coefficient (cf. with the spin $=1 / 2$-chain magnetization modulation in Sec. 6 of Ref. [19]). However, despite such limitations, there is a feature which is consistent with the thresholdless nature of the CB. Figure 9 shows onsite $\mathrm{CB}$ contrast measured for all the system sizes and the interlayer strength studied. The data can be fit by the power-law dependence on $V_{d}, C_{2} \propto V_{d}^{b}, b \approx 4.39$, which is consistent with no threshold in $V_{d}$.

\section{CONCLUSIONS AND OUTLOOK}

Superfluids of hard-core bosons in the multitube geometry turn out to be unstable toward forming composite superfluids, supercounterfluids, and checkerboard insulators for arbitrary small intertube interaction. This conclusion is supported by the bosonization and numerics based on the newly developed ab initio Monte Carlo alghorithm. The hard-core nature of the lattice bosons is the underlying reason for this peculiarity. Filling factors in each tube are the controlling parameters allowing creation of various combinations of these phases. It should also be noted that such flexibility is also due to the 1D dimensionality of the system. As the model simulations [30] have shown, in two-dimensional (2D) layered systems the transition from $N$ atomic to the composite superfluid is of strongly first order for $N \geqslant 3$. We believe this feature should persist in the ab initio simulations. 


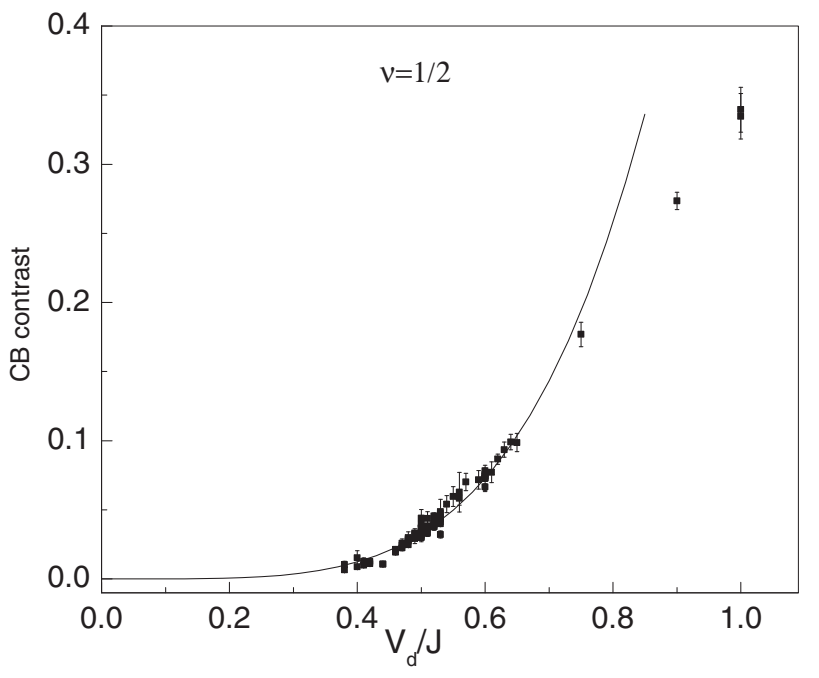

FIG. 9. The CB contrast for $N=3$ plotted for all sizes $L=$ 100-600, in the absence of intralayer repulsion and all interlayer interaction strengths $V_{d} / J$ studied. Solid line is the fit for the power-law dependence $\propto V_{d}^{4.39}$.

In the context of the emergence of parafermions [22] in the multitube geometry with finite intertube tunneling, we find important conducting $a b$ initio simulations of such systems in order to establish the requirements for their experimental realization. In particular, such simulations are needed to infer how the threshold for the transitions depends on the dipolar strength and the tunneling amplitude.

Another interesting system proposed in Refs. [20,21] also requires $a b$ initio simulations for establishing practical ranges of the interaction and lattice parameters. As the bosonization argument indicates, forming a superfluid consisting of complexes of $p \geqslant 1$ hard-core bosons from one tube with $q>1$ such bosons from the other requires exceeding some finite threshold in $V_{d}$. Indeed, the critical value of the Luttinger parameter needed to make the BS harmonic $V_{p,-q}(22)$ relevant is $K_{c}=2 /\left(p^{2}+q^{2}\right)$, which is significantly smaller than $K=$ 1 for the hard-core bosons even for the lowest nontrivial combination $p=1, q=2$. Increasing the intra-layer interaction reduces the $K$ value, so that, potentially, it may be possible to realize the phases $[20,21]$. This, however, needs to be checked by the QMC.

We comment on possibilities of the experimental realization of the phases discussed here. There are two crucial aspects to consider: roles of temperature and of system size. The results obtained above are valid for the corresponding ground states. At finite temperature $T$ in $1 D$ a superfluid loses its asymptotic algebraic order-it transforms into the exponential decay at distances larger than $L_{T} \approx 4 \pi \hbar K V_{s} /\left(K_{B} T\right)$, where $K_{B}$ is Boltzmann constant and $K$ is Luttinger constant. Conversely, in order to observe the algebraic order in a system of size $L$, the temperature must be macroscopically small, that is, $T<T_{L} \approx 4 \pi \hbar K V_{s} /\left(K_{B} L\right)$. This requirement is clearly much more stringent than the condition for observing three-dimensional Bose Einstein condensation characterized by a temperature $T_{\mathrm{BEC}}$ independent of system size.
In the case of the composite phases, say, the composite superfluid there are two energy scales: $T_{\mathrm{CL}} \propto 1 / L$ for the condensation of the composites and $T_{\Delta}$ for their formation. The second one is determined by the binding energy which, at small $V_{d}$ is the exponential function of $V_{d}$, so that $T_{\Delta} \sim$ $J \exp \left(-\cdots J / V_{d}\right)$ with the associated size of the bound state $L_{0} \sim a \exp \left(-\cdots 1 / V_{d}\right)$ (44). It is important to note that, while $T_{\Delta}$ is exponentially supressed for $V_{d} \ll J$, it does not depend on the system size, provided $L>L_{0}$. It should also be mentioned that $T_{\mathrm{CL}}$ is suppressed in addition to that for the individual superfluids by the effective mass enhancement introducing at least the factor of $1 / N$, that is, $T_{\mathrm{CL}} \sim T_{L} / N$.

If the system size is large enough, that is, $L>L_{0}$, then $T_{\Delta}>T_{L}$. Accordingly, lowering $T$ below $T_{\Delta}$ implies the formation of CSF so that no algebraic order will be observed for each individual atomic superfluid at distances $x>L_{0}$. The order in the CSF channel will, however, be observed also only at short distances $x<\sim 1 / T N$. The CSF fully develops only when $T<T_{\mathrm{CL}}$.

Finally, we note that the most optimal condition in terms of the temperature condition should be observed for the $\mathrm{CB}$ insulating states. In this case, there is no continuous broken symmetry so that there is just one energy scale $T_{\Delta}$. Then, lowering $T$ below $T_{\Delta}$ will result in the formation of domains with the $\mathrm{CB}$ order. Their size $L_{D}$ can be estimated from the Landau argument [37] as $L_{D} \sim a \exp \left(T_{\Delta} / T\right)$, where the energy of the CB domain wall is taken as $T_{\Delta}$.

\section{ACKNOWLEDGMENT}

This work was supported by the NSF through a grant to ITAMP at the Harvard-Smithsonian Center for Astrophysics, Grant No. PHY1314469, and by a grant from CUNY HPCC under NSF Grants No. CNS-0855217, No. CNS-0958379, and No. ACI-1126113. This work used the Extreme Science and Engineering Discovery Environment (XSEDE), which is supported by National Science Foundation Grant No. ACI1053575 and the OU Supercomputing Center for Education \& Research (OSCER) at the University of Oklahoma (OU).

\section{APPENDIX A: RG EQUATIONS}

Here we outline derivation of the RG equations for general (weak) interactions. The procedure is a straightforward extension of the standard one (see, e.g., Refs. [19,38]).

For small interactions, the only relevant harmonics in the backscattering terms (22) can be those with the lowest integers $m_{z}, m_{z^{\prime}}$, that is, $m_{z}= \pm 1, m_{z^{\prime}} \pm 1$, with $z \neq z^{\prime}$. The standard renormalization procedure consists of integrating out small oscillations of the Haldane phases $\theta_{z}$ [34] [from the partition function $\left.Z=\int D \theta \exp (-S)\right]$ within the spherical shell of $\vec{q}$ between some cutoff $\Lambda /(1+s)$ and $\Lambda$, and further rescaling $x \rightarrow(1+s) x$ and $\tau \rightarrow(1+s) \tau$, with $s \rightarrow 0$. In the lowest order (one-loop approximation), this procedure implies independent renormalization of each harmonic. Specifically, for the case of $V_{m,-m}$ one finds

$$
\frac{d V_{m ;-m}\left(z, z^{\prime}\right)}{d s}=\left[2-\frac{2 m^{2}}{s}\left\langle\left(\theta_{z}-\theta_{z^{\prime}}\right)^{2}\right\rangle^{\prime}\right] V_{m ;-m}\left(z, z^{\prime}\right)
$$


where $\langle\ldots\rangle^{\prime}$ implies Gaussian average with respect to the action (21), with the integration performed over the shell of the momenta defined above. In the $D=1+1$ dimensions, $\left\langle\left(\theta_{z}-\theta_{z^{\prime}}\right)^{2}\right\rangle^{\prime}$ exhibits log divergence, that is, $\left\langle\left(\theta_{z}-\theta_{z^{\prime}}\right)^{2}\right\rangle^{\prime} \sim$ $\ln (1+s) \sim s$ and it is independent of $\Lambda \rightarrow 0$. Then, the RG flow is controlled by $\ln \Lambda$ or, in a finite system of size $L$, by $\ln L$ so that $d \ldots / d s=d \ldots / d \ln L$.

The mean $\langle\ldots\rangle^{\prime}$ can be represented in terms of the elements of the Luttinger matrix $\hat{K}$ from Eq. (12) which is the inverse of the matrix $\hat{K}^{-1}$ from the dual form (21). Thus, Eq. (A1) becomes

$$
\frac{d V_{m ;-m}\left(z, z^{\prime}\right)}{d s}=\left[2-m^{2}\left(K_{z z}+K_{z^{\prime} z^{\prime}}-2 K_{z z^{\prime}}\right)\right] V_{m ;-m}\left(z, z^{\prime}\right),
$$

where $K_{z z^{\prime}}$ are elements of the matrix $\hat{K}$. In the case of translational invariance along the $z$ axis this equation can be explicitly written in the form (24).

If one ignores the renormalization of the Luttinger matrix, the value of $\tilde{K}\left(q_{z}\right)$ from Eq. (20) can be used. For small $V_{d}$ one can expand Eq. (20) in powers of $V_{d}$ and rewrite Eq. (A2) as

$$
\frac{d V_{m ;-m}(z)}{d s}=\left(2-m^{2}\left\{2 K-\pi K^{2}[\bar{V}(0)-\bar{V}(z)]\right\}\right) V_{m ;-m}(z),
$$

where $\bar{V}(z)$ is given in Eq. (18). In the limit $V_{d} \rightarrow 0$ and for $|m|=1$, the critical value of $K$ is $K_{c}=1$. We also note that higher harmonics $V_{m,-m}(z), m>1$, are irrelevant because the critical value for them in the limit $V_{d} \rightarrow 0$ is $K_{m}=1 / m^{2}<1$.

The renormalization of the BS amplitudes, Eq. (A1), is considered together with the renormalization of the inverse of the matrix $\left(\hat{K}^{-1}\right)_{z z^{\prime}}$ entering the quadratic form (11). In the one-loop approximation the main contribution is due to the same BS harmonic, $\cos \left(2\left(\theta_{z} \pm \theta_{z^{\prime}}\right)\right)$. It generates the term $\sim\left[\vec{\nabla}\left(\theta_{z} \pm \theta_{z^{\prime}}\right)\right]^{2}$ in the second order with respect to the harmonics $\theta^{\prime}$ belonging to the RG shell, where the signs \pm are correlated. Thus, the contributions to the diagonal elements $\left(\hat{K}^{-1}\right)_{z z}$ and to the off-diagonal ones $\left(\hat{K}^{-1}\right)_{z z^{\prime}}$ where $z \neq z^{\prime}$, should be considered independently. Following the standard procedure (see in Refs. $[19,38]$ ), the contribution to $\left(\hat{K}^{-1}\right)_{z z^{\prime}}$ from the BS amplitude $V_{1 ; \pm 1}\left(z, z^{\prime}\right)$, with $z \neq z^{\prime}$, can be represented as

$$
\frac{d\left(\hat{K}^{-1}\right)_{z z^{\prime}}}{d s}= \pm C V_{1 ; \pm 1}^{2}\left(z, z^{\prime}\right) \frac{\left\langle\left(\theta_{z} \pm \theta_{z^{\prime}}\right)^{2}\right\rangle^{\prime}}{s},
$$

where the signs " \pm " are correlated; $C$ is a nonuniversal constant determining type of the short distance cutoff (see in Ref. [19]).

The contributions to $\frac{d\left(\hat{K}^{-1}\right)_{z z}}{d s}$ come from all pairs. Specifically,

$$
\begin{aligned}
\frac{d\left(\hat{K}^{-1}\right)_{z z}}{d s}= & C \sum_{z^{\prime} \neq z}\left[V_{1 ;-1}^{2}\left(z, z^{\prime}\right) \frac{\left\langle\left(\theta_{z}-\theta_{z^{\prime}}\right)^{2}\right\rangle^{\prime}}{s}\right. \\
& \left.+V_{1 ; 1}^{2}\left(z, z^{\prime}\right) \frac{\left\langle\left(\theta_{z}+\theta_{z^{\prime}}\right)^{2}\right\rangle^{\prime}}{s}\right] .
\end{aligned}
$$

Equations (A4) and (A5), where $\langle\ldots\rangle^{\prime}$ implies averaging over the Gaussian fluctuations within the momentum shell, lead to
Eqs. (28), (29), (33), and (34), where it is taken into account that $\pm\left\langle\theta_{z} \theta_{z^{\prime}}\right\rangle^{\prime} \sim \pm s K_{z z^{\prime}}$.

\section{APPENDIX B: RG SOLUTIONS FOR $N=2$}

At $v \neq 1 / 2$ the only relevant harmonic is $V_{1 ;-1}(1)$. Thus the RG flow affects $\tilde{K}(\pi)$ and $V_{1 ;-1}$ only. The corresponding RG equations follow from Eqs. (24), (30), and (31) as

$$
\begin{aligned}
& \frac{d u}{d \ln l}=2(1-g) u, \\
& \frac{d g^{-1}}{d \ln l}=g u^{2},
\end{aligned}
$$

where we used the notations $u=\sqrt{2 C} V_{1 ;-1}(1), g=\tilde{K}(\pi)$. These equations are the standard Kosterlitz-Thoulless [35] RG equations (see in Refs. [19,39]).

The flow $g(l)$ begins at small scales from the initial value set by $g(0)=K / \sqrt{1+\pi K \tilde{V}(\pi)}$, with $\tilde{V}(\pi)=\bar{V}(0)-\bar{V}(1)$. Thus, $g(0)<1$ is below the critical value $K=1$ and the system should become gapped.

The channel $V_{1 ; 1}, \tilde{K}(0)$ is irrelevant as long as $v \neq 1 / 2$. At $v=1 / 2$, or in the case of the complementary filling factors $v_{0}=v$ and $v_{1}=1-v$, the channel $\left[V_{1 ; 1}, \tilde{K}(0)\right]$ must be considered as well. The corresponding RG equations follow from Eqs. (35), (36), and (37) in the same form as Eq. (B1) where now $u=\sqrt{2 C} V_{1 ; 1}(1), g=\tilde{K}(0)$, with the initial value set as $g(0)=K / \sqrt{1+\pi K \tilde{V}(0)}$, with $\tilde{V}(0)=\bar{V}(0)+\bar{V}(1)$. Thus, in the case $N=2$, the channels $V_{1 ; 1}$ and $V_{1 ;-1}$ are decoupled from each other and are described by the same set of equations.

A general solution of the system (B1) can be expressed in terms of two constants of integration, $\eta, l_{0}>0$, determined by the initial values of $u$ and $g$, which in their turn are set by the microscopic model (1). If $\eta$ is real, the solution has a form,

$$
\begin{aligned}
& u^{2}=2\left[\xi^{2}-\eta^{2}\right], \quad \xi=\frac{1}{g}-1, \quad F_{\eta}=4 \ln \left(\frac{l}{l_{0}}\right), \\
& F_{\eta} \equiv \ln \left(\xi^{2}(l)-\eta^{2}\right)+\frac{1}{|\eta|} \ln \left(\frac{\xi(l)-|\eta|}{\xi(l)+|\eta|}\right),
\end{aligned}
$$

where $|\xi|>|\eta|$ and $\xi>-1$. If $\eta=\mathrm{i}|\eta|$, the solution becomes

$$
\begin{aligned}
& u^{2}=2\left[\xi^{2}+|\eta|^{2}\right], \quad \xi=\frac{1}{g}-1, \quad F_{\eta}=4 \ln \left(\frac{l}{l_{0}}\right), \\
& F_{\eta} \equiv \ln \left(\xi^{2}(l)+|\eta|^{2}\right)-\frac{2}{|\eta|} \tan ^{-1}\left(\frac{|\eta|}{\xi}\right),
\end{aligned}
$$

where $\xi>-1$.

The constants $\eta, l_{0}$ are determined by the dipolar interaction $V_{d}$. If $V_{d}=0$, that is, the hard-core bosons are noninteracting (except for the hard-core constraint), the RG equations are trivially satisfied by $\xi=0, u=0$, which implies that $\eta=$ $0, l_{0}=\infty$ for $V_{d}=0$. The critical solution $(\xi=0, u=0)$ belongs to the separatrix, $\eta \rightarrow 0, \xi(l)=\xi_{s}(l), u(l)=u_{s}(l)$ :

$$
u_{s}=\sqrt{2}\left|\xi_{s}\right|, \quad \ln \xi_{s}-\frac{1}{\xi_{s}}=2 \ln \left(\frac{l}{l_{0}}\right) .
$$


Algebraic order exists in the domain $-1<\xi(0)<0, u(0)<$ $u_{s}$, where $\xi$ flows to the stable fixed point $\xi(\infty)=-|\eta|$ for real $\eta$ satisfying $0<|\eta|<1$, and $u(\infty)=0$. All other initial values correspond to the runaway flows $\xi(\infty)=\infty, u(\infty)=$ $\infty$, that is, to the gapped state.

As explicitly shown in Eq. (20), small intertube attractive interaction $V_{d}$ lowers $\tilde{K}(\pi)$ below $K=1$, that is, the initial value of $\xi$ is $\xi(0) \sim\left|V_{d}\right|$. It is also clear that the initial BS interaction $V_{1 ;-1}$ must also be $\sim\left|V_{d}\right|$ in this limit. Thus, $|\eta| \sim$ $\left|V_{d}\right|$, as follows from Eqs. (B2) and (B3).

It is instructive to discuss the dependence $l_{0}$ vs $V_{d}$. As mentioned already, $l_{0}=\infty$ for $V_{d}=0$ and it must become finite as $V_{d} \neq 0$. Thus, $l_{0}$ has a meaning of the correlation length - the size of a dimer forming paired superfluid. The type of dependence can be established from, e.g., Eq. (B3).
Starting from $\xi(0) \sim\left|V_{d}\right|$ at $l \sim 1$, this equation becomes $-\left|V_{d}\right|^{-1} \tan ^{-1}(\kappa) \approx 4 \ln \left(1 / l_{0}\right)$, where $\kappa=|\eta| / \xi(0)$ is some number of the order of unity. Thus,

$$
l_{0} \sim \exp \left(\frac{\kappa^{\prime}}{\left|V_{d}\right|}\right)
$$

where $\kappa^{\prime} \sim 1$. As found in our simulations, Eq. (44), this length, $L_{0}=l_{0}$, determines the properties of the paired superfluid. The dependence (B5) should be, on one hand, contrasted with the temperature divergence $\sim \exp \left(\ldots 1 / \sqrt{T-T_{c}}\right)$ of the correlation length in classical BKT transition on the approach to the critical temperature $T_{c}$ [35], and, on the other, it should be compared with the divergence of the two-body bound state size $\sim \exp \left(\ldots 1 / V_{b}\right)$ in $2 \mathrm{D}$ as the attractive potential $V_{b} \rightarrow 0$.
[1] P. Windpassinger and K. Sengstock, Rep. Prog. Phys. 76, 086401 (2013).

[2] D. Jaksch, C. Bruder, J. I. Cirac, C. W. Gardiner, and P. Zoller, Phys. Rev. Lett. 81, 3108 (1998).

[3] I. Bloch, J. Dalibard, and W. Zwerger, Rev. Mod. Phys. 80, 885 (2008).

[4] A. B. Kuklov and B. V. Svistunov, Phys. Rev. Lett. 90, 100401 (2003).

[5] A. Kuklov, N. Prokofev, and B. Svistunov, Phys. Rev. Lett. 92, 030403 (2004); Ş. G. Söyler, B. Capogrosso-Sansone, N. V. Prokof'ev, and B. V. Svistunov, New J. Phys. 11, 073036 (2009).

[6] D.-W. Wang, M. D. Lukin, and E. Demler, Phys. Rev. Lett. 97, 180413 (2006).

[7] J. Catani, L. De Sarlo, G. Barontini, F. Minardi, and M. Inguscio, Phys. Rev. A 77, 011603 (2008).

[8] B. Gadway, D. Pertot, R. Reimann, and D. Schneble, Phys. Rev. Lett. 105, 045303 (2010).

[9] D. M. Weld, H. Miyake, P. Medley, D. E. Pritchard, and W. Ketterle, Phys. Rev. A 82, 051603 (2010).

[10] J. M. Sage, S. Sainis, T. Bergeman, and David DeMille, Phys. Rev. Lett. 94, 203001 (2005).

[11] K.-K. Ni, S. Ospelkaus, M. H. G. de Miranda, A. Pe'er, B. Neyenhuis, J. J. Zirbel, S. Kotochigova, P. S. Julienne, D. S. Jin, and J. Ye, Science 322, 231 (2008).

[12] J. Deiglmayr, A. Grochola, M. Repp, K. Mörtlbauer, C. Glück, J. Lange, O. Dulieu, R. Wester, and M. Weidemüller, Phys. Rev. Lett. 101, 133004 (2008).

[13] K. Aikawa, D. Akamatsu, M. Hayashi, K. Oasa, J. Kobayashi, P. Naidon, T. Kishimoto, M. Ueda, and S. Inouye, Phys. Rev. Lett. 105, 203001 (2010).

[14] S. Ospelkaus, K.-K. Ni, D. Wang, M. H. G. de Miranda, B. Neyenhuis, G. Quéméner, P. S. Julienne, J. L. Bohn, D. S. Jin, and J. Ye, Science 327, 853 (2010).

[15] T. Takekoshi, M. Debatin, R. Rameshan, F. Ferlaino, R. Grimm, H. C. Nägerl, C. R. Le Sueur, J. M. Hutson, P. S. Julienne, S. Kotochigova, and E. Tiemann, Phys. Rev. A 85, 032506 (2012).

[16] C.-H. Wu, J. W. Park, P. Ahmadi, S. Will, and Martin W. Zwierlein, Phys. Rev. Lett. 109, 085301 (2012).

[17] M. Repp, R. Pires, J. Ulmanis, R. Heck, E. D. Kuhnle, M. Weidemüller, and E. Tiemann, Phys. Rev. A 87, 010701 (2013).
[18] B. Yan, S. A. Moses, B. Gadway, J. P. Covey, K. R. A. Hazzard, A. M. Rey, D. S. Jin, and J. Ye, Nature 501, 521 (2013).

[19] T. Giamarchi, Quantum Physics in One Dimension (Oxford University Press, Oxford, 2004).

[20] E. Burovski, G. Orso, and T. Jolicoeur, Phys. Rev. Lett. 103, 215301 (2009).

[21] B. Wunsch, N. T. Zinner, I. B. Mekhov, S.-J. Huang, D.-W. Wang, and E. Demler, Phys. Rev. Lett. 107, 073201 (2011).

[22] A. M. Tsvelik and A. B. Kuklov, New J. Phys. 14, 115033 (2012).

[23] P. Lecheminant and H. Nonne, Phys. Rev. B 85, 195121 (2012).

[24] G. E. Astrakharchik, G. De Chiara, G. Morigi, and J. Boronat, J. Phys. B 42, 154026 (2009).

[25] M. Klawunn, J. Duhme, and L. Santos, Phys. Rev. A 81, 013604 (2010).

[26] A. C. Potter, E. Berg, D.-W. Wang, B. I. Halperin, and E. Demler, Phys. Rev. Lett. 105, 220406 (2010).

[27] M. A. Baranov, A. Micheli, S. Ronen, and P. Zoller, Phys. Rev. A 83, 043602 (2011).

[28] J. R. Armstrong, N. T. Zinner, D. V. Fedorov, and A. S. Jensen, Eur. Phys. J. D 66, 85 (2012).

[29] D. Hufnagl and R. E. Zillich, Phys. Rev. A 87, 033624 (2013).

[30] B. Capogrosso-Sansone and A. Kuklov, J. Low Temp. Phys. 165, 213 (2011).

[31] N. V. Prokof'ev, B. V. Svistunov, and I. S. Tupitsyn, Phys. Lett. A 238, 253 (1998); Sov. Phys. JETP 87, 310 (1998).

[32] L. Mathey, Phys. Rev. B 75, 144510 (2007).

[33] E. L. Pollock and D. M. Ceperley, Phys. Rev. B 36, 8343 (1987).

[34] F. D. M. Haldane, Phys. Rev. Lett. 45, 1358 (1980).

[35] J. M. Kosterlitz and D. J. Thouless, J. Phys.: C Solid State Phys. 6, 1181 (1973).

[36] N. Prokofev and B. Svistunov, Phys. Rev. A 66, 043608 (2002).

[37] L. D. Landau and E. M. Lifshitz, in Statistical Physics, Part 1: Volume 5. Course of Theoretical Physics, 3rd ed. (ButterworthHeinemann, Oxford, 2000), p. 537.

[38] M. A. Cazalilla, A. F. Ho, and T. Giamarchi, NJP 8, 158 (2006).

[39] T. C. Lubensky and P. M. Chaikin, Principles of Condensed Matter Physics (Cambridge University Press, Cambridge, 2000). 